Review Article

\title{
Obesity Animal Models for Acupuncture and Related Therapy Research Studies
}

\author{
Xuwen Zhang $\mathbb{D}^{1,2,3}$ and David Val-Laillet $\mathbb{D}^{3}$ \\ ${ }^{1}$ Guangzhou University of Chinese Medicine, Guangzhou, China \\ ${ }^{2}$ Panyu Central Hospital, Guangzhou, China \\ ${ }^{3}$ INRAE, INSERM, Univ Rennes, Nutrition Metabolisms and Cancer, NuMeCan, Rennes, St Gilles, France \\ Correspondence should be addressed to David Val-Laillet; david.val-laillet@inrae.fr
}

Received 5 November 2020; Accepted 2 September 2021; Published 30 September 2021

Academic Editor: Ihsan Ul Haq

Copyright (c) 2021 Xuwen Zhang and David Val-Laillet. This is an open access article distributed under the Creative Commons Attribution License, which permits unrestricted use, distribution, and reproduction in any medium, provided the original work is properly cited.

\begin{abstract}
Obesity and related diseases are considered as pandemic representing a worldwide threat for health. Animal models are critical to validate the effects and understand the mechanisms related to classical or innovative preventive and therapeutic strategies. It is, therefore, important to identify the best animal models for translational research, using different evaluation criteria such as the face, construct, and predictive validity. Because the pharmacological treatments and surgical interventions currently used for treating obesity often present many undesirable side effects, relatively high relapse probabilities, acupuncture, electroacupuncture (EA), and related therapies have gained more popularity and attention. Many kinds of experimental animal models have been used for obesity research studies, but in the context of acupuncture, most of the studies were performed in rodent obesity models. Though, are these obesity rodent models really the best for acupuncture or related therapies research studies? In this study, we review different obesity animal models that have been used over the past 10 years for acupuncture and EA research studies. We present their respective advantages, disadvantages, and specific constraints. With the development of research on acupuncture and EA and the increasing interest regarding these approaches, proper animal models are critical for preclinical studies aiming at developing future clinical trials in the human. The aim of the present study is to provide researchers with information and guidance related to the preclinical models that are currently available to investigate the outcomes of acupuncture and related therapies.
\end{abstract}

\section{Introduction}

Obesity, which is now considered as a pandemic, is highly prevalent in America and European countries. According to the 2016 data from the WHO, 36.2\% of adults in America were obese, $22.3 \%$ in Germany, and $21.6 \%$ in France [1], whereas $18 \%$ of children and adolescents worldwide were considered as overweight or obese. Obesity, which is basically defined as a pathological increase in bodyweight and fat mass, is also related to many diseases such as type- 2 diabetes, cardiovascular diseases [2], subfertility [3], bone microarchitecture [4], and even to some forms of cancer [5]. Obesity is also frequently associated with eating disorders and psychological problems that complicate the clinical picture and increase the risk for further health problems. The cost and energy to treat these diseases represent a huge financial and societal burden. There is still no consensus treatment recognized as effective and completely safe. Acupuncture and electroacupuncture (EA) are gaining more and more attention for their health outcomes and little adverse effects, though research exploring the outcomes of these therapies cannot be performed exclusively in humans because of ethics and practical constraints and because mechanistic studies are required, with the necessity to sample biological tissues when necessary. Some factors such as diet and physical exercise are also much easier to control in animal models compared to human volunteers.

Obesity is a multifactorial and complex disease involving metabolic disorders, neurohormonal-altered processes affecting many organs such as the gut, liver, and brain, as well 
as pathological behavioral features, and quite often a lowgrade chronic inflammation. There are many kinds of animal models that have been used for obesity research, including rodent models such as mice and rats [6], as well as large animals such as the pig, dog, sheep, macaque, and other nonhuman primate species [7] and even nonmammalian models such as the zebrafish [8] and drosophila [9]. The ways to induce obesity models (referring to their construction validity) are also various, such as high-fat and/or high-sugar diet exposure, spontaneous mutation exploitation or genetic engineering, and iatrogenic induction [10]. The most frequently used obesity animal models for acupuncture and EA research studies still are the rodent models. They are very interesting models to investigate specific physiological or metabolic features of obesity and the way they can be affected by potential treatments. Because rodent gut-brain anatomy and functioning are very different from those in humans and because the analogy with behavioral and cognitive features of obesity in humans is better achieved with closer species in terms of ontogeny, large animal models should appear more relevant to study these particular questions. When it comes to localizing acupuncture points, it is obvious that analogy with humans is better achieved in large animal species that are morphologically and/or ontogenetically closer to the human.

The localization of acupuncture points is associated with specific anatomical and physiological features. Many acupuncture points are in the vicinity of major nerves, blood, or lymphatic vessels. These locations are richly innervated and have autonomic nervous associations. Such locations include nerve penetration of fascia, exits through bony foramina, neurovascular bundles, and sites of nerve branching [11]. Additionally, many acupuncture point locations are also associated with regions that generate muscular dysfunction and pain, such as myofascial trigger points or musculotendinous junctions and muscle motor points. For acupuncture and EA, it is critical to use obese animals that have relatively suitable body volumes to enable researchers to distinguish different muscles, bones, and specific anatomic locations. In this study, we will use the Chinese names to identify the acupoints of interest and will indicate the World Health Organization (WHO) corresponding codes in brackets. For animals, specific names and codes are used according to $\mathrm{Yu}$ [12], and we will indicate them in addition to their human equivalents if necessary.

Some points are located on the basis of anatomical landmarks, some of which presenting similarities or analogies with human points. Other points are pain points (also known as trigger points or Ashi points). In contrast to acupuncture points, pain points have no defined position. They usually occur near lesions but sometimes arise quite far away from them. Most clinicians locate the pain points by systematic pressure palpation of the spine and the paraspinal and limb musculature or stimulate the skin of the affected area and surroundings to locate pain points using a medical reflex hammer [13]. Ashi points are also located on pain or tension areas and are revealed by the avoidance reactions elicited by palpation [14]. Different from humans, animals cannot tell veterinarians where they feel pain, which requires alternative strategies to locate Ashi points. Reflex hammer or Von Frey hair, for example, can be used to investigate the animals' response to stimulation in the vicinity of painful areas, but patience and experience are needed from the veterinarians to interpret these behavioral responses.

The aim of this review is to provide some comparative data for the use of acupuncture, electroacupuncture, and related therapies in the human and different animal models including rodents, nonhuman primates, and large animal models such as pigs, in the context of obesity and related diseases. We will present the respective advantages, disadvantages, and specific constraints of these models compared to humans in relation to their face, construct, and predictive validity. Finally, research perspectives will be proposed with the aim to identify which scientific questions should be investigated in the human or in animal models, respectively, to understand how acupuncture and related therapies can be applied in clinics and which underlying mechanisms are involved.

\section{Human Studies on Obesity and Acupuncture/Electroacupuncture}

Obesity derives from a wide range of physiopathological factors in the scope of biological, socioeconomic, and cultural influences. These factors can include numerous polymorphic gene products, psychosocial, and behavioral factors, food choice, gut microbiome, and chronobiology. Obesity can be the result of a pathological process or pharmacological treatment, but it is also a risk factor for the development of many comorbid conditions [15], such as hypertension and cardiovascular morbidity. It is often accompanied by many alterations at the hormonal, inflammatory, and endothelial levels, also including anomalies of the sympathetic nervous system, renal function, and at the microvascular level. Insulin resistance is a factor that can stimulate and potentiate other pathological mechanisms [16].

Food intake control and consequently bodyweight management depend on homeostatic regulations and on complex behavioral and neurocognitive processes that take place in specific brain regions involved, respectively, in the hedonic dimension of food intake and its cognitive control. Modulating the activity of these brain structures represents a promising strategy to improve food intake control [17]. Latest approaches for obesity treatment focus on medicine targets in the central nervous system (e.g., the leptin-melanocortin axis, the opioid system, GLP-1/GLP-1 system, and FGF21/FGFR1c/b-Klotho axis), but only a few investigational agents will be able to meet the FDA regulatory criteria and advance onto the marketplace [18]. Other strategies are explored, such as nonpharmacological approaches (e.g., microbiome replacement or supplementation), but all these strategies have modest effects, and their long-term safety and adverse effects need to be further investigated [19]. The exploration of safe therapies, as an alternative or in combination with other treatment strategies, is consequently needed.

Acupuncture, electroacupuncture (EA), and related therapies have been widely used to treat obesity in clinics (Table 1). Related research studies have reported positive 
TABLE 1: Overview of studies on human acupuncture and related therapies in the context of obesity.

\begin{tabular}{|c|c|c|c|c|}
\hline Subjects & $\begin{array}{l}\text { Treatment types and sham/control } \\
\text { design }\end{array}$ & $\begin{array}{l}\text { Acupuncture points and } \\
\text { time frequency }\end{array}$ & Main results & References \\
\hline \multicolumn{5}{|c|}{ Acupuncture and electroacupuncture } \\
\hline $\begin{array}{l}\text { RCT, randomized, } \\
\text { patient- and assessor- } \\
\text { blind, sham-controlled } \\
\text { clinical trial, women } \\
\text { aged } \geq 19 \text {, BMI } \\
\geq 25 \mathrm{~kg} / \mathrm{m}^{2}\end{array}$ & $\begin{array}{l}\text { Real group }(N=48) \text { : manual } \\
\text { acupuncture }(\mathrm{MA})+\text { EA vs. the } \\
\text { control group }(N=43) \text { : sham } \\
\text { MA + sham EA sham MA: } \\
\text { nonpenetrating acupuncture at } \\
\text { traditional acupuncture points. } \\
\text { Sham EA: acupuncture at sites not } \\
\text { corresponding to traditional } \\
\text { acupuncture points with } \\
\text { penetration to the same depth but } \\
\text { no electrical stimulation }\end{array}$ & $\begin{array}{l}\text { Body points: Hegu (LI4), } \\
\text { Quchi (LI11), Sanyinjiao } \\
\text { (SP6), Zusanli (ST36), } \\
\text { Qihai (CV6), Zhongwan } \\
\text { (CV12), Tianshu (ST25), } \\
\text { and Shuidao (ST28). Time } \\
\text { and frequency: twice per } \\
\text { week for } 6 \text { weeks }\end{array}$ & $\begin{array}{l}\text { MA and EA treatment did } \\
\text { not affect anthropometric } \\
\text { and serum metabolic } \\
\text { parameters, but increased } \\
\text { carnitine levels (C2, C4, C6, } \\
\text { and L-carnitine) }\end{array}$ & $\begin{array}{l}\text { Kim et al., } \\
2020[20]\end{array}$ \\
\hline $\begin{array}{l}\text { RCT, single-blind, } \\
\text { overweight women, } \\
\text { aged } 20-30, \mathrm{BMI} \geq 25\end{array}$ & $\begin{array}{l}\text { AA group }(N=30) \text { : auricular } \\
\text { acupuncture vs. the sham group } \\
(N=28) \text { : Similar plaster as the AA } \\
\text { group without needle attached }\end{array}$ & $\begin{array}{l}\text { Auricular points: } \\
\text { Shenmen (TF4) and } \\
\text { appetite control (TG2). } \\
\text { Time and frequency: } 20 \\
\text { minutes per time, once a } \\
\text { week for totally } 7 \text { weeks }\end{array}$ & $\begin{array}{l}\text { AA treatment reduced waist } \\
\text { circumference, but weight } \\
\text { loss and BMI were not } \\
\text { significant in both groups: } \\
\text { have the effect on mood } \\
\text { improvement }\end{array}$ & $\begin{array}{l}\text { Lillingston } \\
\text { et al., } 2019 \\
{[21]}\end{array}$ \\
\hline $\begin{array}{l}\text { RCT double-blind, obese } \\
\text { adolescents, BMI 25-30 }\end{array}$ & $\begin{array}{l}\text { Auricular group }(N=32) \text { : auricular } \\
\text { acupressure at obesity effective } \\
\text { points with Vaccaria seeds vs. the } \\
\text { sham group }(N=26) \text { : placebo } \\
\text { auricular acupressure and } \\
\text { noneffective points on obesity }\end{array}$ & $\begin{array}{l}\text { Auricular points: auricular } \\
\text { group, Shenmen (TF4), } \\
\text { spleen (CO13), endocrine } \\
\text { (CO18), stomach (CO4), } \\
\text { and hunger (TG1); control } \\
\text { group zone has no } \\
\text { relationship with obesity, } \\
\text { knee zone, hip-joint zone, } \\
\text { lumber zone, thoracic- } \\
\text { vertebrae zone, and tooth } \\
\text { zone time and frequency. } \\
\text { Both } 8 \text { weeks, one time } \\
\text { one week, } 5 \text { minutes } \\
\text { pressure one time before } \\
\text { meals and whenever they } \\
\text { felt hungry; ears were } \\
\text { treated alternately }\end{array}$ & $\begin{array}{l}\text { Auricular acupressure was } \\
\text { better than the control } \\
\text { group: decreased TC and } \\
\text { LDL-C levels }\end{array}$ & $\begin{array}{l}\text { Cha and } \\
\text { Park, } 2019 \\
\quad[22]\end{array}$ \\
\hline $\begin{array}{l}\text { RCT, male, aged }>60 \text {, } \\
\text { with sarcopenic obesity }\end{array}$ & $\begin{array}{c}\text { EA + AA group }(N=23) \text { : EA + oral } \\
\text { essential amino acids vs. the AA } \\
\text { group }(N=25) \text { : oral essential amino } \\
\text { acids alone }\end{array}$ & $\begin{array}{l}\text { Body points: Binao (LI14), } \\
\text { Quchi (LI11), Biguan } \\
\text { (ST31), and Liangqiu } \\
\text { (ST34). Time and } \\
\text { frequency: } 20 \text { minutes per } \\
\text { time, once every } 3 \text { days for } \\
12 \text { weeks; essential amino } \\
\text { acids orally, twice per day } \\
\text { for } 28 \text { weeks }\end{array}$ & $\begin{array}{l}\text { Both decreased BFP and } \\
\text { increased ASM/H2; } \\
\text { EA + AA is more effective } \\
\text { and can increase muscle } \\
\text { mass in a shorter time }\end{array}$ & $\begin{array}{l}\text { Zhou et al., } \\
2018 \text { [23] }\end{array}$ \\
\hline $\begin{array}{l}\text { RCT, abdominal obese } \\
\text { women, aged } 21-53 \text {, } \\
\mathrm{BMI} \geq 23 \text {, WC }>80 \mathrm{~cm}\end{array}$ & $\begin{array}{l}\text { EA group }(N=15) \text { : body points vs. } \\
\text { the control group }(N=15) \text { : no } \\
\text { intervention + maintained normal } \\
\text { diet + usual exercise habits }\end{array}$ & $\begin{array}{l}\text { Body points: Huaroumen } \\
\text { (ST24), Shuidao (ST28), } \\
\text { Huaroumen (ST24), } \\
\text { Shuidao (ST28), Fujie } \\
\text { (SP14), and Daheng } \\
\text { (SP15). Time and } \\
\text { frequency: three times a } \\
\text { week for } 3 \text { months }\end{array}$ & $\begin{array}{c}\text { EA treatment reduces BMI } \\
\text { and WC as well as VAT } \\
\text { volume and HFF }\end{array}$ & $\begin{array}{l}\text { Lei et al., } \\
2017 \text { [24] }\end{array}$ \\
\hline
\end{tabular}


TABle 1: Continued.

\begin{tabular}{|c|c|c|c|c|}
\hline Subjects & $\begin{array}{l}\text { Treatment types and sham/control } \\
\text { design }\end{array}$ & $\begin{array}{l}\text { Acupuncture points and } \\
\text { time frequency }\end{array}$ & Main results & References \\
\hline $\begin{array}{l}\text { RCT, overweight and } \\
\text { obese subjects, BMI } \geq 25\end{array}$ & $\begin{array}{c}\text { EA group }(N=79) \text { : body } \\
\text { points + low-calorie diet vs. the } \\
\text { control group }(N=82) \text { : low-calorie } \\
\text { diet }\end{array}$ & $\begin{array}{c}\text { Body points: Tianshu } \\
\text { (ST25), Weidao (GB28), } \\
\text { bilateral, Zhongwan } \\
\text { (CV12), Shuifen (CV9), } \\
\text { Guanyuan (CV4), } \\
\text { Sanyinjiao (SP6), Quchi } \\
\text { (LI11), Fenglong (ST40), } \\
\text { Qihai (CV6), and } \\
\text { Yinlingquan (SP9). Time } \\
\text { and frequency: } 20 \text { minutes } \\
\text { each time, two treatment } \\
\text { sessions per week for } 6 \\
\text { weeks + follow up } 6 \text { weeks }\end{array}$ & $\begin{array}{c}\text { EA is better than low- } \\
\text { calorie diet alone, serum } \\
\text { prooxidant antioxidant } \\
\text { balance, and reduces serum } \\
\text { PAB values }\end{array}$ & $\begin{array}{l}\text { Mazidi et al., } \\
2017 \text { [25] }\end{array}$ \\
\hline $\begin{array}{l}\text { RCT, obese women, } \\
\text { BMI } 30-40\end{array}$ & $\begin{array}{l}\text { Body group }(N=21) \text { : receive } \\
\text { acupuncture at body acupoints vs. } \\
\text { the auricular group }(N=17) \text { : receive } \\
\text { acupuncture at auricular acupoints }\end{array}$ & $\begin{array}{c}\text { Auricular points: } \\
\text { antiaggression (nicotine } \\
\text { addiction points), } \\
\text { stomach (CO4); body } \\
\text { points: Hegu (L14), Quchi } \\
\text { (LIll), Tianshu (ST25), } \\
\text { Zusanli (ST36), Sanyinjiao } \\
\text { (SP6), Yinlingquan (SP9), } \\
\text { Zhongwan (CV12), and } \\
\text { Qihai (CV6). Time and } \\
\text { frequency: auricular } \\
\text { group: } 6 \text { sessions in every } \\
\text { 15 days, manual } \\
\text { stimulation of all four } \\
\text { needles 15-20 minutes } \\
\text { before meals for } 30 \\
\text { seconds and } 3 \text { times one } \\
\text { day; body group: two }\end{array}$ & $\begin{array}{l}\text { Both groups: changes in } \\
\text { weight, body mass index, } \\
\text { body fat percentage, waist } \\
\text { circumference, and hip } \\
\text { circumference were } \\
\text { statistically significant; } \\
\text { auricular acupuncture is } \\
\text { more effective in reducing } \\
\text { bodyweight than body } \\
\text { acupuncture }\end{array}$ & $\begin{array}{l}\text { Yasemin } \\
\text { et al., 2017 } \\
{[26]}\end{array}$ \\
\hline $\begin{array}{l}\text { RCT, double-blind, } \\
\text { overweight/obese } \\
\text { T2DM adult patients, } \\
\text { aged } 20-65, \text { BMI } \geq 25\end{array}$ & $\begin{array}{c}\text { EA group }(\mathrm{N}=19) \text { : body } \\
\text { EA + auricular points } \\
\text { pressure + metformin vs. the sham } \\
\text { group }(N=20) \text { : sham body } \\
\text { EA + sham auricular points } \\
\text { pressure + metformin sham design: } \\
\text { maximally superficial needles on } \\
0.3 \mathrm{~cm} \text { laterally from acupoints and } \\
\text { adhesive tape without seeds on } \\
\text { auricular points }\end{array}$ & $\begin{array}{l}\text { sessions, } 12 \text { weeks } \\
\text { Body points: Zhongwan } \\
\text { (CV12), Tianshu (ST25), } \\
\text { Zusanli (ST36), Sanyinjiao } \\
\text { (SP6), Shuifen (REN9), } \\
\text { Hegu (LI4), Daheng } \\
\text { (SP15), Shuidao (ST28), } \\
\text { Guanyuan (REN4), Quchi } \\
\text { (LI11), and Qihai (REN6). } \\
\text { Auricular points: Sanjiao } \\
\text { (CO17), hunger (TG1), } \\
\text { stomach (CO4), Shenmen } \\
\text { (TF4), endocrine (CO18), } \\
\text { and spleen (CO13). Time } \\
\text { and frequency: both 10 } \\
\text { times, } 30 \text { minutes every } \\
\text { time, every other day, } 3 \\
\text { weeks; auricular points } \\
\text { were treated } 2 \text { ears } \\
\text { alternately }\end{array}$ & $\begin{array}{l}\text { EA was better than sham } \\
\text { EA, decreased BW, BMI, } \\
\text { FBS, FINS, HOMA-IR, IL- } \\
\text { 6, TNF- } \alpha \text {, leptin, GLP-1, } \\
\text { resistin, ceramides, FFA, } \\
\text { TG, and LDL-C and } \\
\text { increased HDL-C, } \\
\text { adiponectin, and serotonin }\end{array}$ & $\begin{array}{l}\text { Firouzjaei } \\
\text { et al., } 2016 \\
{[27]}\end{array}$ \\
\hline
\end{tabular}


TABle 1: Continued.

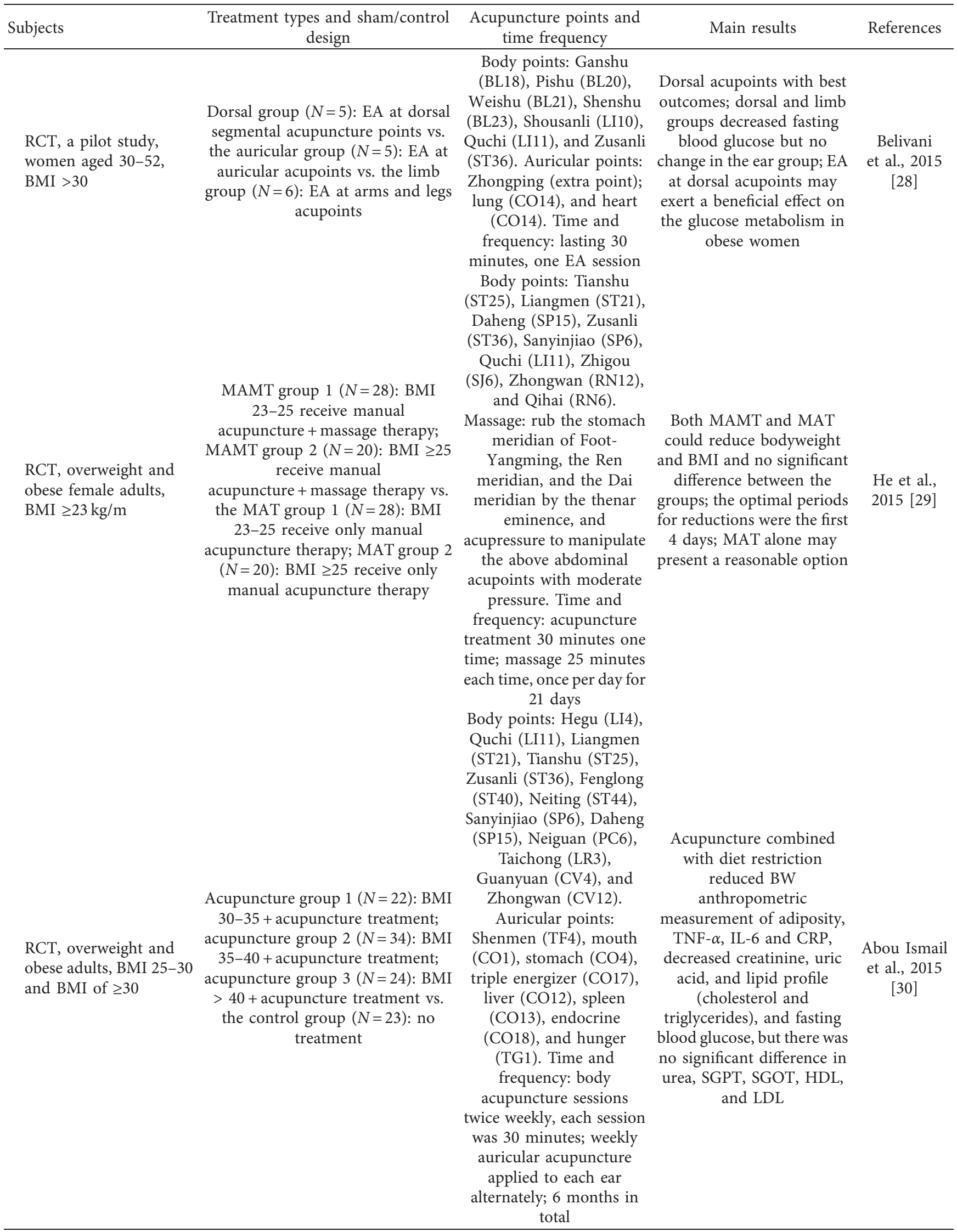


TABle 1: Continued.

\begin{tabular}{|c|c|c|c|c|}
\hline Subjects & $\begin{array}{l}\text { Treatment types and sham/control } \\
\text { design }\end{array}$ & $\begin{array}{l}\text { Acupuncture points and } \\
\text { time frequency }\end{array}$ & Main results & References \\
\hline $\begin{array}{l}\text { RCT, single-blinded, } \\
\text { adults aged } 18-50, \mathrm{BMI} \\
\geq 27\end{array}$ & $\begin{array}{l}\text { EA group }(N=36) \text { : both auricular } \\
\text { electrical stimulation with } \\
\text { electrodes + acupressure with seed- } \\
\text { embedding vs. the sham group } \\
(N=34) \text { : points not associated with } \\
\text { weight reduction or obesity }\end{array}$ & $\begin{array}{c}\text { Auricular points: EA } \\
\text { group: Shenmen (TF4), } \\
\text { stomach (CO4), endocrine } \\
\text { (CO18), and hunger } \\
\text { (TG1); sham group: ankle } \\
\text { (AH3), elbow (SF3), } \\
\text { shoulder (SF4), and } \\
\text { clavicle (SF6). Time and } \\
\text { frequency: EA once a week } \\
\text { for } 20 \text { minutes, } \\
\text { acupressure } 1 \text { min, } 4 \text { times } \\
\text { per day, } 10 \text { weeks in total; } \\
\text { ears were treated } \\
\text { alternatively }\end{array}$ & $\begin{array}{l}\text { No significant difference } \\
\text { between } 2 \text { groups; EA } \\
\text { group: TC, TG, and leptin } \\
\text { decreased after } \\
\text { intervention; sham group: } \\
\text { TC, leptin decreased, and } \\
\text { adiponectin increased after } \\
\text { intervention }\end{array}$ & $\begin{array}{l}\text { Yeh et al., } \\
2015[31]\end{array}$ \\
\hline $\begin{array}{l}\text { Randomized crossover } \\
\text { pilot study, single- } \\
\text { blinded, overweight and } \\
\text { obese adults, BMI } 25-40\end{array}$ & $\begin{array}{l}\text { Acupuncture group }(N=19) \text { : } \\
\text { nutritional } \\
\text { counseling + acupuncture vs. the } \\
\text { sham group ( } N=16) \text { : nutritional } \\
\text { counseling + sham acupuncture } \\
\text { (sites were located close to the } \\
\text { classical acupuncture points) }\end{array}$ & $\begin{array}{l}\text { Body acupoints: Hegu } \\
\text { (LI4), Quchi (LI11), } \\
\text { Zusanli (ST356), Neiting } \\
\text { (ST44), and Taichong } \\
\text { (LR3). Auricular } \\
\text { acupoints: hunger (TG1), } \\
\text { stomach (CO4), and } \\
\text { Shenmen (TF4). Time and } \\
\text { frequency: } 30 \text { minutes } \\
\text { each time, twice weekly for } \\
6 \text { weeks, with a 2-week } \\
\text { wash-out period }\end{array}$ & $\begin{array}{l}\text { A larger trial investigating } \\
\text { the use of acupuncture for } \\
\text { weight loss in those who } \\
\text { have elevated eating and } \\
\text { weight concerns is feasible }\end{array}$ & $\begin{array}{l}\text { Fogarty } \\
\text { et al., } 2015 \\
{[32]}\end{array}$ \\
\hline $\begin{array}{l}\text { RCT, adults, age } 18-65 \text {, } \\
\text { BMI } \geq 25 \text {, male waist line } \\
\geq 90 \mathrm{~cm} \text {, female waist } \\
\text { line } \geq 80 \mathrm{~cm} \text {, abdominal } \\
\text { obesity with spleen } \\
\text { deficiency and } \\
\text { exuberant dampness }\end{array}$ & $\begin{array}{l}\text { Channel group }(N=33) \text { : } \\
\text { acupuncture at hour-prescriptive } \\
\text { points from } 9 \text { to } 11 \text { AM vs. the } \\
\text { control group }(N=32) \text { : acupuncture } \\
\text { at any time beyond } 9-11 \text { AM }\end{array}$ & $\begin{array}{c}\text { Body acupoints: Fujie } \\
\text { (SP14), Daheng (SP15), } \\
\text { Xuehai (SP10), } \\
\text { Yinlingquan (SP9), Diji } \\
\text { (SP8), Sanyinjiao (SP6), } \\
\text { and Gongsun (SP4). Time } \\
\text { and frequency: } 30 \\
\text { minutes, once every day } \\
\text { for three courses of } \\
\text { treatment with ten } \\
\text { sessions }\end{array}$ & $\begin{array}{l}\text { The total curative effect in } \\
\text { the channel group was } \\
\text { better than in the control } \\
\text { group in reducing BW, } \\
\text { BMI, waistline, obesity } \\
\text { level, and clinical } \\
\text { symptoms; there was no } \\
\text { significant difference in } \\
\text { WHR between the } 2 \text { groups }\end{array}$ & $\begin{array}{l}\text { Wu et al., } \\
2014 \text { [33] }\end{array}$ \\
\hline $\begin{array}{l}\text { RCT, single-blinded, } \\
\text { aged } 18-55 \text {, abdomen } \\
\text { fat mass obesity, man, } \\
\text { BMI } 0-40\end{array}$ & $\begin{array}{l}\text { Body EA group }(N=20) \text { : low-calorie } \\
\text { diet + body EA vs. the auricular EA } \\
\text { group }(N=20) \text { : low-calorie } \\
\text { diet + auricular acupuncture } \\
\text { (auricular pressing plasters with } \\
\text { seeds) vs. the sham body group } \\
(N=20) \text { : low-calorie diet + sham } \\
\text { body EA: superficial inserts } 0.5 \mathrm{cun} \\
\text { cranially and } 0.5 \text { cun laterally from } \\
\text { acupoints vs. the sham auricular } \\
\text { group }(N=20) \text { : low-calorie } \\
\text { diet + sham auricular acupuncture, } \\
\text { auricular plasters without seeds }\end{array}$ & $\begin{array}{l}\text { Body acupoints: Tianshu } \\
\text { (ST25), Weidao (GB28), } \\
\text { Zhongwan (REN12), } \\
\text { Shuifen (REN9), } \\
\text { Guanyuan (REN4), and } \\
\text { Sanyinjiao (SP6). } \\
\text { Auricular points: } \\
\text { Shenmen (TF4), stomach } \\
\text { (CO4), hunger (TG1), } \\
\text { mouth (CO1), center of } \\
\text { auricular (HX1), and } \\
\text { triple energizer (CO17); } \\
\text { ears were treated } \\
\text { alternately; time and } \\
\text { frequency: body EA 20 } \\
\text { minutes one time; } \\
\text { auricular plaster kept } \\
\text { attached } 3 \text { days one time, } \\
\text { both twice a week, } 6 \text { weeks } \\
\text { in total }\end{array}$ & $\begin{array}{c}\text { Both EA groups: WC and } \\
\text { HC were reduced } \\
\text { postintervention; compared } \\
\text { with sham groups, } \\
\text { decreased BMI, TFM, WC, } \\
\text { and HC body in the EA } \\
\text { group: more effective on } \\
\text { WC; auricular EA group: } \\
\text { more effective on HC }\end{array}$ & $\begin{array}{c}\text { Darbandi } \\
\text { et al., } 2014 \\
{[34]}\end{array}$ \\
\hline
\end{tabular}


TABle 1: Continued.

\begin{tabular}{|c|c|c|c|c|}
\hline Subjects & $\begin{array}{l}\text { Treatment types and sham/control } \\
\text { design }\end{array}$ & $\begin{array}{l}\text { Acupuncture points and } \\
\text { time frequency }\end{array}$ & Main results & References \\
\hline $\begin{array}{l}\mathrm{RCT}, \text { female } \\
\text { overweight/obese } \\
\text { students, aged } 20-30, \\
\mathrm{BMI} \geq 25\end{array}$ & $\begin{array}{c}\text { Auricular acupressure group } \\
(N=25) \text { : auricular acupoints } \\
\text { pressure with Sinapsis alba seeds vs. } \\
\text { the control group }(N=24) \text { : no } \\
\text { intervention }\end{array}$ & $\begin{array}{c}\text { Auricular points: } \\
\text { Shenmen (TF4), mouth } \\
\text { (CO1), stomach (CO4), } \\
\text { endocrine (CO18), and } \\
\text { small intestine (CO6). } \\
\text { Time and frequency: press } \\
\text { those points } 10 \text { times at a } \\
\text { rate of two times per } \\
\text { second, } 30 \text { minutes before } \\
\text { mealtime, three times } \\
\text { daily for one month; ears } \\
\text { were treated alternately }\end{array}$ & $\begin{array}{c}\text { Auricular acupoints } \\
\text { pressure decreased BW and } \\
\text { BMI and increased self- } \\
\text { efficacy }\end{array}$ & $\begin{array}{l}\text { Kim et al., } \\
2014 \text { [35] }\end{array}$ \\
\hline $\begin{array}{l}\text { RCT, single blind, obese } \\
\text { adults, aged } \geq 19, \text { BMI } \\
\geq 23\end{array}$ & $\begin{array}{l}\text { 5-point group }(N=22) \text { : auricular } \\
\text { acupuncture with indwelling } \\
\text { needles at } 5 \text {-point vs. the } 1 \text {-point } \\
\text { group }(\mathrm{N}=21) \text { : auricular } \\
\text { acupuncture with indwelling } \\
\text { needles only at hunger point vs. the } \\
\text { sham group }(N=15) \text { : needles fixed } \\
\text { on surgical tape, needles removed } \\
\text { immediately after insertion } 2 \text { mm at } \\
\text { the } 5 \text { points selected for the } 5 \text {-point } \\
\text { group, and surgical tape remained }\end{array}$ & $\begin{array}{l}\text { Auricular acupoints: 5- } \\
\text { point group, Shenmen } \\
\text { (TF4), spleen (CO13), } \\
\text { stomach (CO4), hunger } \\
\text { (TG1), and endocrine } \\
\text { (CO18); 1-point group: } \\
\text { hunger point; only time } \\
\text { and frequency: one time } \\
\text { attach last one week, once } \\
\text { a week for } 8 \text { weeks; ears } \\
\text { were treated alternately } \\
\text { Auricular points: hunger }\end{array}$ & $\begin{array}{l}\text { Both auricular acupuncture } \\
\text { treatment groups reduce } \\
\text { BMI, BW, and BF mass } \\
\text { compared to the sham } \\
\text { group; the } 5 \text {-point group } \\
\text { reduced BMI by } 6.1 \% \text { and } \\
\text { the 1-point group by } 5.7 \%\end{array}$ & $\begin{array}{l}\text { Yeo et al., } \\
2014[36]\end{array}$ \\
\hline $\begin{array}{l}\text { RCT, randomized } \\
\text { placebo-controlled, } \\
\text { pilot, double-blinded } \\
\text { study, female obese } \\
\text { adults, aged }>18, \text { BMI } \\
>25\end{array}$ & $\begin{array}{c}\text { Verum group }(N=28) \text { : auricular EA } \\
\text { with P-Stim }{ }^{\circledR} \text { device vs. the sham } \\
\text { group }(N=28) \text { : P-Stim }{ }^{\circledR} \text { dummy } \\
\text { device had no power supply and had } \\
\text { been grinded to leave only metal } \\
\text { plates }\end{array}$ & $\begin{array}{l}\text { (TG1) and stomach } \\
\text { (CO4). Time and } \\
\text { frequency: four days in } \\
\text { one week, for a period of } \\
\text { six weeks. A follow-up } \\
\text { visit was performed after } 4 \\
\text { weeks; ears were treated } \\
\text { alternately }\end{array}$ & $\begin{array}{l}\text { Auricular EA better than } \\
\text { the placebo group, with a } \\
\text { decrease of BW and BMI }\end{array}$ & $\begin{array}{c}\text { Schukro } \\
\text { et al., } 2014 \\
{[37]}\end{array}$ \\
\hline $\begin{array}{l}\text { RCT, overweight and } \\
\text { obese adults, aged } \\
18-55 \text {, BMI } 25-45\end{array}$ & $\begin{array}{l}\text { Auricular group }(N=86) \text { : inserted } \\
\text { the auricular pressing plaster with } \\
\text { seed + low-calorie diet vs. the sham } \\
\text { group }(N=83) \text { : inserted the } \\
\text { auricular pressing plaster without } \\
\text { seed on points with no relationship } \\
\text { with obesity }\end{array}$ & $\begin{array}{l}\text { Auricular acupoints: } \\
\text { auricula group: Shenmen } \\
\text { (TF4), stomach (CO4), } \\
\text { hunger (TG1), mouth } \\
\text { (CO1), center of auricular } \\
\text { (HX1), and triple } \\
\text { energizer (CO17); sham } \\
\text { group: hip (AH5), spleen } \\
\text { (CO13), nose (TG3), and } \\
\text { esophagus (CO2). Time } \\
\text { and frequency: pressure to } \\
\text { the auricular points } 30 \\
\text { minutes before eating for } \\
\text { about } 20 \text { seconds; seed } \\
\text { plasters were changed } \\
\text { twice a week for a total of } 6 \\
\text { weeks; ears were treated } \\
\text { alternately }\end{array}$ & $\begin{array}{l}\text { Auricular group was better: } \\
\text { reduced anthropometric } \\
\text { factors and anti-Hsp } \\
\text { antibodies }\end{array}$ & $\begin{array}{l}\text { Abdi et al., } \\
2012[38]\end{array}$ \\
\hline $\begin{array}{l}\text { RCT, randomized, } \\
\text { sham-controlled } \\
\text { preliminary trial, female } \\
\text { adults, BMI }>30\end{array}$ & $\begin{array}{l}\text { Acupuncture group }(N=20) \text { : body } \\
\text { points vs. the sham group }(N=20) \text { : } \\
\text { acupuncture needles were not } \\
\text { inserted but just applied under a } \\
\text { tape at the same points }\end{array}$ & $\begin{array}{l}\text { Body acupoints: Hegu } \\
\text { (LI4), Shenmen (HT7), } \\
\text { Zusanli (ST36), Neiting } \\
\text { (ST44), and Sanyinjiao } \\
\text { (SP6); time and frequency: } \\
\text { two sessions of } 20 \text { min per } \\
\text { week for a total } 5 \text { weeks }\end{array}$ & $\begin{array}{c}\text { Decreased insulin and } \\
\text { leptin levels, BW, and BMI; } \\
\text { increased plasma ghrelin } \\
\text { and CCK compared with } \\
\text { sham }\end{array}$ & $\begin{array}{c}\text { Gucel et al., } \\
2012 \text { [39] }\end{array}$ \\
\hline
\end{tabular}


TABle 1: Continued.

\begin{tabular}{|c|c|c|c|c|}
\hline Subjects & $\begin{array}{l}\text { Treatment types and sham/control } \\
\text { design }\end{array}$ & $\begin{array}{l}\text { Acupuncture points and } \\
\text { time frequency }\end{array}$ & Main results & References \\
\hline $\begin{array}{l}\text { RCT, overweight and } \\
\text { obese adults, aged } \\
18-55 \text {, BMI } 25-40\end{array}$ & $\begin{array}{l}\text { Acupuncture group }(N=79) \text { : } \\
\text { acupuncture + low-calorie diet vs. } \\
\text { the sham group }(N=82) \text { : low- } \\
\text { calorie diet }+ \text { sham, superficial } \\
\text { needle at nonacupoints } 0.5 \mathrm{~cm} \text { up } \\
\text { and } 0.5 \mathrm{~cm} \text { laterally to the real } \\
\text { acupoints }\end{array}$ & $\begin{array}{c}\text { Body acupoints: Tianshu } \\
\text { (ST25), Weidao (GB28), } \\
\text { Zhongwan (REN12), } \\
\text { Shuifen (REN9) } \\
\text { Guanyuan (REN4), } \\
\text { Sanyinjiao (SP6), Quchi } \\
\text { (LI11), Fenglong (ST40), } \\
\text { Qihai (REN6), and } \\
\text { Yinlingqau (SP9). Time } \\
\text { and frequency: 20 minutes } \\
\text { each time, two sessions per } \\
\text { week for a total of } 6 \\
\text { weeks, + second period of } \\
6 \text { weeks only low-calorie } \\
\text { diet and exercise for all } \\
\text { participants }\end{array}$ & $\begin{array}{l}\text { Anti-Hsp-antibodies only } \\
\text { decreased in the } \\
\text { acupuncture group; } \\
\text { changes in lipid profiles } \\
\text { were observed in the } \\
\text { control group only; } \\
\text { acupuncture combination } \\
\text { with diet restriction was } \\
\text { effective in enhancing } \\
\text { weight loss and improving } \\
\text { dyslipidemia }\end{array}$ & $\begin{array}{l}\text { Abdi et al., } \\
2012[38]\end{array}$ \\
\hline $\begin{array}{l}\text { RCT, young adults, aged } \\
18-20, \mathrm{WC} \geq 80 \mathrm{~cm} \text { in } \\
\text { females and } \geq 90 \mathrm{~cm} \text { in } \\
\text { males }\end{array}$ & $\begin{array}{c}\text { Control group }(N=28) \text { : Only } \\
\text { acupressure vs. the experimental } \\
\text { group }(N=27) \text { : } \\
\text { acupressure + Japanese Magnetic } \\
\text { Pearl on the ear acupoints }\end{array}$ & $\begin{array}{l}\text { Auricular acupoints: } \\
\text { Shenmen (TF4), mouth } \\
\text { (CO1), stomach (CO4), } \\
\text { small intestine (CO6), and } \\
\text { endocrine (CO18). Time } \\
\text { and frequency: once } \\
\text { weekly for ten minutes for } \\
\text { a total of eight weeks } \\
\text { Auricular acupoints: }\end{array}$ & $\begin{array}{l}\text { Both groups decreased BW } \\
\text { and WC; only the } \\
\text { experimental group with } \\
\text { Japanese Magnetic Pearls } \\
\text { decreased WHR }\end{array}$ & $\begin{array}{l}\text { Hsieh et al., } \\
2011[40]\end{array}$ \\
\hline $\begin{array}{l}\text { RCT, overweight } \\
\text { adolescents, aged } 18-20 \text {, } \\
\text { BMI } \geq 23\end{array}$ & $\begin{array}{c}\text { Vaccaria seeds group }(N=26) \text { : } \\
\text { auricular acupressure with Vaccaria } \\
\text { seeds vs. the JMP group }(N=24) \text { : } \\
\text { auricular acupoints stimulation with } \\
\text { Japanese magnetic tap vs. the } \\
\text { control group }(N=26) \text { : nothing on } \\
\text { tape }\end{array}$ & $\begin{array}{l}\text { Shenmen (TF4), mouth } \\
\text { (CO1), stomach (CO4), } \\
\text { endocrine (CO18), and } \\
\text { small intestine (CO6). } \\
\text { Time and frequency: ears } \\
\text { were treated alternately; } \\
\text { the tape was replaced } \\
\text { every two or three days; 8- } \\
\text { week study }\end{array}$ & $\begin{array}{l}\text { Both auricular acupuncture } \\
\text { treatments decreased BMI; } \\
\text { Vaccaria seeds provided } \\
\text { better outcomes; all three } \\
\text { groups increased TC, TG, } \\
\text { HDL-C, and LDL-C }\end{array}$ & $\begin{array}{l}\text { Hsieh, } 2010 \\
\quad[41]\end{array}$ \\
\hline $\begin{array}{l}\text { RCT, postmenopausal } \\
\text { women, aged }<64 \text {, } \\
\text { percentage of body fat: } \\
>30 \% \text {, waist } \\
\text { circumference }>80 \mathrm{~cm}\end{array}$ & $\begin{array}{l}\text { EAS group }(N=20) \text { : stimulation at } \\
\text { acupoint by a modulated middle- } \\
\text { frequency interferential current } \\
\text { electrical device vs. the control } \\
\text { group }(N=21) \text { : no treatment }\end{array}$ & $\begin{array}{l}\text { Body acupoints: Zusanli } \\
\text { (ST36) and Sanyinjiao } \\
\text { (SP6). Time and } \\
\text { frequency: } 20 \text { minutes } \\
\text { each time, twice a week for } \\
12 \text { consecutive weeks }\end{array}$ & $\begin{array}{l}\text { Middle-frequency current } \\
\text { electrical stimulation at } \\
\text { acupoints decreased body } \\
\text { composition (weight, waist, } \\
\text { and hip circumference, } \\
\text { percentage of body fat, and } \\
\text { percentage of lean muscle } \\
\text { mass) }\end{array}$ & $\begin{array}{l}\text { Lin et al., } \\
2010[42]\end{array}$ \\
\hline $\begin{array}{l}\text { RCT, single blind, obese } \\
\text { females, aged 16-65, } \\
\text { BMI }>27\end{array}$ & $\begin{array}{l}\text { Auricular group }(N=23) \text { : received } \\
\text { auricular acupuncture vs. the sham } \\
\text { group }(N=22) \text { : received placebo } \\
\text { needles on nonacupoint }\end{array}$ & $\begin{array}{l}\text { Auricular acupoints: } \\
\text { hunger (TG1), Shenmen } \\
\text { (TF4), stomach (CO4), } \\
\text { and endocrine (CO18). } \\
\text { Time and frequency: } \\
\text { needles kept on auricular } 3 \\
\text { days with no pressure; two } \\
\text { treatments per week for a } \\
\text { total of } 6 \text { weeks with } 12 \\
\text { treatments; ears were } \\
\text { treated alternately }\end{array}$ & $\begin{array}{c}\text { Auricular group increased } \\
\text { ghrelin and decreased } \\
\text { leptin levels }\end{array}$ & $\begin{array}{l}\text { Hsu et al., } \\
2009[43]\end{array}$ \\
\hline
\end{tabular}


TABle 1: Continued.

\begin{tabular}{|c|c|c|c|c|}
\hline Subjects & $\begin{array}{l}\text { Treatment types and sham/control } \\
\text { design }\end{array}$ & $\begin{array}{l}\text { Acupuncture points and } \\
\text { time frequency }\end{array}$ & Main results & References \\
\hline $\begin{array}{l}\text { Randomized crossover } \\
\text { trial, } 46 \text { simple obese } \\
\text { females, aged } 16-65 \text {, } \\
\text { BMI }>30 \text {, WC }>90 \mathrm{~cm}\end{array}$ & $\begin{array}{l}\text { EA group }(N=22) \text { : body points vs. } \\
\text { the sit-up exercises group }(N=20) \text { : } \\
\text { sit-up exercise vs. the control group } \\
\qquad(N=21) \text { : no intervention }\end{array}$ & $\begin{array}{l}\text { Body acupoints: Qihai } \\
\text { (CV6), Shuifen (CV9), } \\
\text { Shuidao (ST28), Siman } \\
\text { (K14), Zusanli (ST26), } \\
\text { Fenglong (ST40), and } \\
\text { Sanginjao (SP6). Time and } \\
\text { frequency: EA } 40 \text { minutes } \\
\text { each time, two treatments } \\
\text { per week; sit-up exercises: } \\
10 \text { times per day, } 6 \text { weeks } \\
\text { in total } \\
\text { Body acupoints: Qihai } \\
\text { (CV6), Shuifen (CV9), } \\
\text { Shuidao (ST28), Siman } \\
\text { (K14), Zusanli (ST26), } \\
\text { Fenglong (ST40), and } \\
\text { Sanginjao (SP6). Time and } \\
\text { frequency: EA twice a } \\
\text { week; sit-up exercise 10 } \\
\text { times per day, } 6 \text { weeks in } \\
\text { total }\end{array}$ & $\begin{array}{c}\text { EA group was better than } \\
\text { the two other groups in } \\
\text { decreasing BW, BMI, and } \\
\text { WC }\end{array}$ & $\begin{array}{l}\text { Hsu et al., } \\
2005[44]\end{array}$ \\
\hline \multicolumn{5}{|c|}{ Acupoints embedding, laser acupuncture, or moxibustion } \\
\hline $\begin{array}{l}\text { RCT, double blinded, } \\
\text { adults, aged } 18-60 \text {, } \\
\text { BMI }>25 \text {, abdominal } \\
\text { circumferences }>80 \mathrm{~cm} \\
\text { in women and }>90 \mathrm{~cm} \text { in } \\
\text { men }\end{array}$ & $\begin{array}{l}\text { Laser acupuncture group }(N=19) \text { : } \\
\text { laser acupuncture + dietary } \\
\text { intervention vs. the sham group } \\
\quad(N=19) \text { : sham laser } \\
\text { acupuncture + dietary intervention }\end{array}$ & $\begin{array}{l}\text { Body points: Fenglong } \\
\text { (ST40), Tianshu (ST25), } \\
\text { Sanyinjiao (SP6), Zusanli } \\
\text { (ST36), and Zhongwan } \\
\text { (CV12). Time and } \\
\text { frequency: } 3 \text { times a week } \\
\text { for } 4 \text { weeks }\end{array}$ & $\begin{array}{l}\text { Laser acupuncture had } \\
\text { good effects on WHR, QoL } \\
\text { scores, BMI, and appetite } \\
\text { scores in obese patients }\end{array}$ & $\begin{array}{c}\text { Sebayang } \\
\text { et al., } 2020 \\
{[46]}\end{array}$ \\
\hline $\begin{array}{l}\text { RCT, patient-assessor- } \\
\text { blinded, randomized, } \\
\text { sham-controlled } \\
\text { crossover trial, adults } \\
\text { aged }>20 \text {, BMI }>25\end{array}$ & $\begin{array}{l}\text { Laser acupuncture group }(N=26) \text { : } \\
\text { laser applied by a GaAlAs } \\
\text { semiconductor diode laser } \\
\text { phototherapy device vs. the sham } \\
\text { group }(N=26) \text { : placebo treatment } \\
\text { under the laser with no power } \\
\text { output; the same physician } \\
\text { performed all laser applications }\end{array}$ & $\begin{array}{l}\text { Body points: Tianshu } \\
\text { (ST25), Zusanli (ST36), } \\
\text { Fenglong (ST40), Neiting } \\
\text { (ST44), Hegu (LI4), Quchi } \\
\text { (LI11), Sanyinjiao (SP6), } \\
\text { and Neiguan (PC6). Time } \\
\text { and frequency: three times } \\
\text { a week for } 8 \text { weeks; after a } \\
\text { two-week washout period, } \\
\text { the subjects received the } \\
\text { treatment of the opposite } \\
\text { group for another } 8 \text { weeks }\end{array}$ & $\begin{array}{l}\text { Laser acupuncture } \\
\text { decreased BMI, body fat } \\
\text { percentage, WHR, waist } \\
\text { circumference, and hip } \\
\text { circumference compared to } \\
\text { sham; significantly } \\
\text { improved scores on the } \\
\text { fullness, hunger, satiety, } \\
\text { desire to eat, and overall } \\
\text { well-being relative to the } \\
\text { baseline }\end{array}$ & $\begin{array}{l}\text { Tseng et al., } \\
2016 \text { [47] }\end{array}$ \\
\hline
\end{tabular}


TABle 1: Continued.

\begin{tabular}{|c|c|c|c|c|}
\hline Subjects & $\begin{array}{l}\text { Treatment types and sham/control } \\
\text { design }\end{array}$ & $\begin{array}{l}\text { Acupuncture points and } \\
\text { time frequency }\end{array}$ & Main results & References \\
\hline $\begin{array}{l}\text { RCT, adults, aged } \\
25-42, \mathrm{BMI} \geq 28, \mathrm{WHR} \\
>0.9 \text { in men, WHR } \\
>0.85 \text { in women, WtHR } \\
>0.5\end{array}$ & $\begin{array}{l}\text { Polyglycolic acid sutures embedding } \\
\text { therapy (PASET) group }(N=28) \text { : } \\
\text { PASET alternatively in abdominal I } \\
\text { group and II group vs. the control } \\
\text { group }(N=23) \text { : lifestyle } \\
\text { modification }\end{array}$ & $\begin{array}{c}\text { Body acupoints: (I) } \\
\text { Zhongwan (CV12), } \\
\text { Shuifen (CV9), Qihai } \\
\text { (CV6), Fuliu (CV7), } \\
\text { Shuiguan (CV5), } \\
\text { Mangshu (KI16), Siman } \\
\text { (KI14), Taiyi (ST23), } \\
\text { Tianshu (ST25), Daju } \\
\text { (ST27), Fujie (SP14), } \\
\text { Daheng (SP15), and } \\
\text { Daimai (GB26); (II) Jianli } \\
\text { (RN11), Xiawan (RN10), } \\
\text { Guanyuan (RN4), Shiguan } \\
\text { (KI18), Shangqu (KI17), } \\
\text { Zhongzhu (KI15), Qixue } \\
\text { (KI13), Huaroumen } \\
\text { (ST24), Wailing (ST26), } \\
\text { Shuidao (ST28), Daheng } \\
\text { (SP15), Fuai (SP160), and } \\
\text { Daimai (GB26). Time and } \\
\text { frequency: the treatment } \\
\text { cycle was repeated every } \\
\text { 10 days, 10 weeks }\end{array}$ & $\begin{array}{l}\text { PASET was better than } \\
\text { lifestyle change; PASET } \\
\text { significantly reduced BW, } \\
\text { BMI, hip circumference, } \\
\text { waist circumference, WHR, } \\
\text { WtHR, and thickness of } \\
\text { abdominal subcutaneous } \\
\text { fat tissue; PASET also } \\
\text { improved the evaluated } \\
\text { scores in aspects of physical } \\
\text { function, self-esteem, } \\
\text { public distress, and sexual } \\
\text { life, as well as decreased } \\
\text { blood pressure, glycemia, } \\
\text { low-density lipoprotein, } \\
\text { uric acid, and the levels of } \\
\text { tumor necrosis factor- } \\
\text { alpha, interleukin-1 } \beta \text {, and } \\
\text { increased high density } \\
\text { lipoprotein; lifestyle } \\
\text { modification only } \\
\text { illustrated a trend for } \\
\text { weight decrease }\end{array}$ & $\begin{array}{l}\text { Chen et al., } \\
2019 \text { [48] }\end{array}$ \\
\hline $\begin{array}{l}\text { RCT, double-blind, } \\
\text { placebo-controlled, } \\
\text { women, BMI } \geq 27 \text {, WC } \\
\geq 80 \mathrm{~cm} \text {, aged } 20-65\end{array}$ & $\begin{array}{l}\text { Catgut embedding group }(N=45) \text { : } \\
\text { receive acupoint catgut embedding } \\
\text { vs. the sham group }(N=45) \text { : the } \\
\text { embedding acupoints, treatment } \\
\text { frequency, and duration were the } \\
\text { same, but without chromic catgut } \\
\text { strands }\end{array}$ & $\begin{array}{l}\text { Body acupoints: Qihai } \\
\text { (CV6), Shuifen (CV9), } \\
\text { Shuidao (ST28), Siman } \\
\text { (KI14), and Zusanli } \\
\text { (ST36). Time and } \\
\text { frequency: once a week, } 6 \\
\text { weeks in total }\end{array}$ & $\begin{array}{l}\text { Catgut embedding better } \\
\text { reduced } \mathrm{BW}, \mathrm{WC} \text {, leptin to } \\
\text { adiponectin ratio, and } \\
\text { improved leptin resistance }\end{array}$ & $\begin{array}{l}\text { Chen et al., } \\
2019 \text { [48] }\end{array}$ \\
\hline $\begin{array}{l}\text { RCT, overweight or } \\
\text { obese female adults, } \\
\text { BMI } \geq 25\end{array}$ & $\begin{array}{l}\text { ACET with the moxibustion group } \\
(N=19) \text { : acupuncture catgut } \\
\text { embedding + moxibustion vs. the } \\
\text { sham group }(N=18) \text { : using } \\
\text { stainless-steel needles covered with } \\
\text { a plastic film and a cap to avoid } \\
\text { needle insertion }\end{array}$ & $\begin{array}{l}\text { Body points: Qihai (CV6), } \\
\text { Zhongwan (CV12), } \\
\text { Tianshu (ST25), Zusanli } \\
\text { (ST36), Sanyinjiao (SP6), } \\
\text { Pishu (BL20), and } \\
\text { Shenshu (BL23). Time and } \\
\text { frequency: catgut was } \\
\text { implanted every } 3 \text { weeks; } \\
5 \text { min moxibustion was } \\
\text { applied twice a week for a } \\
\text { total of } 6 \text { weeks }\end{array}$ & $\begin{array}{l}\text { ACET with moxibustion } \\
\text { did not modify circulating } \\
\text { adipokines levels; } \\
\text { transcriptional changes in } \\
\text { adipose tissue revealed } \\
\text { modulation of genes } \\
\text { participating in } \\
\text { homeostasis control, lipid } \\
\text { metabolism, olfactory } \\
\text { transduction, and gamma- } \\
\text { aminobutyric acid signaling } \\
\text { pathway }\end{array}$ & $\begin{array}{c}\text { Garcia-Vivas } \\
\text { et al., } 2016 \\
{[49]}\end{array}$ \\
\hline $\begin{array}{l}\text { RCT, } 66 \text { women, aged } \\
>20 \text { with postpartum } \\
\text { weight retention, } \\
\text { postpartum duration }<1 \\
\text { month, BMI }>25\end{array}$ & $\begin{array}{l}\text { Laser acupuncture group }(N=33) \text { : } \\
\text { using gallium aluminum arsenide } \\
\text { laser pen vs. the sham group } \\
(N=33) \text { : same acupoints without } \\
\text { any laser output }\end{array}$ & $\begin{array}{l}\text { Body acupoints: Tianshu } \\
\text { (ST25), Shuidao (ST28), } \\
\text { Fenglong (ST40), Daheng } \\
\text { (SP15), Shuifen (CV9), } \\
\text { and Sanyinjiao (SP6). } \\
\text { Auricular points: stomach } \\
\text { (CO4) and hunger points } \\
\text { (TG1). Time and } \\
\text { frequency: } 12 \text { treatment } \\
\text { sessions; } 5 \text { times a week, } \\
\text { approximately } 3 \text { weeks }\end{array}$ & $\begin{array}{l}\text { Laser acupuncture reduced } \\
\text { postpartum weight } \\
\text { retention by improving } \\
\text { BMI and BFP; no effect on } \\
\text { WB }\end{array}$ & $\begin{array}{l}\text { Hung et al., } \\
2016 \text { [50] }\end{array}$ \\
\hline
\end{tabular}


TABle 1: Continued.

\begin{tabular}{|c|c|c|c|c|}
\hline Subjects & $\begin{array}{l}\text { Treatment types and sham/control } \\
\text { design }\end{array}$ & $\begin{array}{l}\text { Acupuncture points and } \\
\text { time frequency }\end{array}$ & Main results & References \\
\hline $\begin{array}{l}\text { RCT, randomized, } \\
\text { placebo-controlled, } \\
\text { single blind, adults, aged } \\
18-45 \text {, BMI } \geq 25\end{array}$ & $\begin{array}{l}\text { AM group }(N=22) \text { : acupuncture } \\
\text { with moxibustion vs. the LNAM } \\
\text { group }(N=10) \text { : long needle } \\
\text { acupuncture with moxibustion vs. } \\
\text { the EA group }(N=10) \text { : EA only vs. } \\
\text { the EAM group }(N=20) \text { : EA with } \\
\text { moxibustion vs. the CGM group } \\
(N=25) \text { : catgut embedding with } \\
\text { moxibustion vs. the sham group } \\
(N=12) \text { : using stainless-steel } \\
\text { needles covered with a plastic film } \\
\text { and a cap to avoid needle insertion }\end{array}$ & $\begin{array}{l}\text { Body acupoints: Qihai } \\
\text { (CV6), Zhongwan } \\
\text { (CV12), Tianshu (ST25), } \\
\text { Zusanli (ST36), Sanyinjiao } \\
\text { (SP6), Pishu (BL20), and } \\
\text { Shenshu (BL23). Time and } \\
\text { frequency: AM, LNAM, } \\
\text { EA, EAM, and sham } \\
\text { groups received two } \\
\text { treatments per week for a } \\
\text { total of } 6 \text { weeks } \\
\text { (acupuncture for } 20 \text { min } \\
\text { with or without } \\
\text { moxibustion for } 5 \text { min in } \\
\text { each session); for the } \\
\text { CGM group, catgut was } \\
\text { implanted every } 3 \text { weeks, } \\
\text { for a total of } 6 \text { weeks, } \\
\text { whereas moxibustion was } \\
\text { applied twice a week }\end{array}$ & $\begin{array}{l}\text { Acupoint catgut embedding } \\
\text { therapy combined with } \\
\text { moxibustion reduced BW, } \\
\text { BMI, insulin, and HOMA- } \\
\text { IR and improved insulin } \\
\text { sensitivity }\end{array}$ & $\begin{array}{c}\text { Garcia-Vivas } \\
\text { et al., } 2014 \\
{[51]}\end{array}$ \\
\hline
\end{tabular}

Not RCT studies presenting brain activity data

Acute stimulation: EA $(N=26,9$ male) and sham ( $N=19,4$ male $)$ group (needles were inserted

Acute stimulation: 45 overweight or obese, 32 superficially to a depth of less than females and 13 males; Long-term treatment: 32 overweight or obese, 9 males and 23 females

\section{$5 \mathrm{~mm}$ and $2 \mathrm{~cm}$ adjacent to the} selected acupoints without manipulation). Long-term treatment: $\mathrm{EA}(N=17,5$ males) and sham $(N=15,4$ males) group (same as acute stimulation)
19 right-handed overweight males aged 21-45, BMI 18-30
Body acupoints: Zhongwan (CV12), Xiawan (CV10), Tianshu (ST25), Daheng (SP15), Liangmen (ST21), Huaroumen (ST24), Wailing (ST26), Daju (ST27), and Fujie (SP14). Additional distribution acupoints: Shangjuxu (ST37), Xiajuxu (ST39), Quchi (LI11), Neiting (ST44), Zhigou (TE6), Hegu (LI4), and Fenglong (ST40). Time and frequency: acute stimulation: only once, needles in body 30 minutes, manipulation needles for 1 minute at every 15 minutes; longer time treatments: 32 minutes each time, three times a week, 6-8 weeks Body acupoints: Zusanli (ST36) and Yinlingquan (SP9). Time and

Acute acupuncture group $(N=10)$ : body points vs. the sham group $(N=9)$ : inserted needles superficially and immediately removed them but pretended to rotate frequency: rotated needles

2 minutes at a rate of 60 times per minute; needles were kept in the body during 21 minutes
Acute stimulation: both groups: weaker positive RSFC between insula and SMA/right dorsolateralprefrontal-cortex (DLPFC) and weaker negative RSFC between insula and precuneus, stronger negative RSFC between DLPFC and DMPFC.

Longer time treatments: EA group: higher BMI reduction, resting activity, and RSFC implicated in inhibitory control, gastric motility, and satiety control are associated with EAinduced weight-loss

Increased hypothalamushippocampus FC; decreased hypothalamusthalamus FC
Ren et al., 2020 [52] 
outcomes, with or without concomitant interventions in terms of diet and physical activity. Based on effective evidence of acupuncture treatment on human drug/alcohol addiction [54-56], Chen et al. [57] proposed a potential treatment role for food addiction and obesity since drug addiction presents evident similarities with food addiction [58]. Although, there is no human or animal study addressing specifically the effectiveness of acupuncture in treating food addiction, many studies have supported the use of acupuncture or EA for obesity treatment. The mechanisms are still unclear, but neuroendocrine regulation may play an important role [59].

Previous research studies reported different cerebral response patterns following the stimulation of acupuncture points in different spinal segments. Zusanli/Sanyinjiao (ST36/SP6) stimulation in healthy human subjects activated the orbitofrontal cortex and deactivated the hippocampus, while Yinlingquan/Chengshan (GB34/BL57) activated the dorsal thalamus and inhibited the primary motor area and premotor cortex [60]. In healthy right-handed women, acupuncture at Zusanli (ST36) increased the anterior insula hemodynamic signal and decreased that in limbic and paralimbic structures including the amygdala, anterior hippocampus, and the subgenual and retrosplenial cingulate cortices, while only EA activated the anterior middle cingulate cortex [61]. In healthy right-handed adults, acupuncture at Hegu (LI4) and Zusanli (ST36) increased brain activity in the hypothalamus and nucleus accumbens, while deactivating the rostral part of anterior cingulate cortex, amygdala formation, and hippocampal complex, in comparison to a sham procedure consisting in superficial pricking on nonacupuncture points on the leg [62]. We can notice that Zusanli (ST36) is a classic point in acupuncture and EA but the outcomes depend on the type of patients and protocols.

Research studies were also performed in subjects with bodyweight management problems. In overweight humans, acupuncture on Yinlingquan (SP9) and Zusanli (ST36) decreased the hypothalamus-thalamus functional connectivity, which was negatively correlated with hunger. It was suggested that the increased dopamine modulation during acupuncture was possibly associated with the decreased poststimulation limbic system and spinothalamic tract connectivity. This correlation was absent under sham stimulation with a similar procedure where needles were removed immediately [53]. More recently, Ren et al. [52] investigated in overweight/obese adults the acute and longterm (6-8 weeks) brain responses to acute EA and sham stimulation (superficially to a depth less than $5 \mathrm{~mm}$ and $2 \mathrm{~cm}$ adjacent to the selected acupoints). They found significant differences between groups in terms of resting-state brain activity and functional connectivity, in the acute and longterm conditions, and in brain areas involved in the inhibitory, hedonic, and/or cognitive control of food intake, gastric motility, and satiety control (e.g., dorsolateral and ventrolateral prefrontal cortex, orbitofrontal cortex, insula, and precuneus). EA treatment was also associated with significant weight loss. Although these results are very interesting, it is important to specify that 16 acupuncture points (distributed on the abdomen, legs, and arms) were used in combination, which makes impossible to discuss their respective roles and importance for obesity treatment. Despite the clinical value of these data, they are not sufficient to describe and support the mechanisms underlying the observed outcomes. Very few studies demonstrated the effects of acupuncture and related therapies on the nervous system and especially brain functioning. Clinical trials are facing strict ethics and recruitment constraints, which makes difficult the comparison between different strategies in terms of acupoints combination or stimulation mode. This is why preclinical studies in relevant animal models are needed.

\section{Rodent Models of Obesity and Acupuncture/Electroacupuncture}

Rodent models present many anatomical and physiological differences compared with humans; however, we cannot ignore their wide use in obesity research and remarkable inputs to biomedical research in general. First, there are many kinds of obesity rodent models, including genetically engineered models and diet-induced models based on the consumption of high-energy diets that are very often highfat diets. Second, rodent models are cheaper and easier to use for animal experimentation than large animals and nonhuman primates. Regulations and ethical rules framing rodent experimentation are also less restrictive that those framing the use of nonhuman primates. Eventhough rats and mice are rather close in terms of phylogeny, they present many differences (e.g., technical considerations, basic functional differences, social, addictive, and impulsive behaviors) leading to potentially different experimental outcomes [63], which need to be considered in the context of acupuncture and related therapies research.

From a "construction validity" point of view, diet-induced obesity is the most natural way to mimic modern obesity epidemic in developed countries, which mainly takes its roots in the chronic consumption of high-energy palatable foods. Swiss mice [64], C57BL/6NCrl mice [65], and C57Bla6J mice [66] can easily declare obesity after about 10-19 weeks of high-calorie diet. Sprague-Dawley rats fed with a high-fructose diet during 8 weeks showed some metabolic disturbances similar to what is described in human obesity, e.g., ectopic lipid deposition, altered hepatic insulin sensitivity, and increased de novo lipogenesis [67]. In Wistar rats, the metabolic effects of a high-energy diet are more pronounced and detected earlier than in Sprague-Dawley rats. These effects include increased weight gain, body fat mass, mesenteric adipocytes' size, adiponectin, and leptin plasma levels, as well as decreased oral glucose tolerance [68]. The gut microbiota of these obese rats also presented more abundant Bacteroides and Prevotella taxa, but less Bifidobacterium and Lactobacillus compared to SD rats, which may represent an important vector of metabolic disturbances [68], considering the role of the microbiotagut-brain axis in the emergence of metabolic diseases [69]. The offspring born from pregnant SD rats, $50 \%$ food restricted from gestational day 10 to term, initially presented abnormal lipogenesis before declaring obesity during the 
period of catch-up growth [70]. The adipose tissue is the first site to exhibit increased de novo synthesis and desaturase activity [70].

Obesity rodent models can also be achieved by specific genetic crossing. Spontaneously hypertensive stroke-prone SHRSP/IDmcr-fas rat is established by crossing Izumo SHRSP rats (SHRSP/Izm) with Zucker fatty (fa/fa) rats. These rats exhibit multiple obesity risk factors, including severe hypertension, obesity, and hyperlipidemia [71]. Otsuka Long-Evans Tokushima Fatty (OLETF) rat is an early onset overeatinginduced obesity for spontaneous mutation, characterized by the lack of expression of functional CCK1 [72]. Zucker rats are hyperphagic, especially towards high-fat food [73], and have lower levels of locomotor activity [73], which mimics the sedentary habits usually observed in human obese subjects. They have mutated leptin receptor genes [74], i.e., two homozygous fa/fa "fatty" alleles resulting in leptin insensitivity, which is related to hyperphagic phenotype and higher appetite [75]. KK/HlJ (KK inbred strain was inbred by Kuo Kondo in 1944. The KK/HlJ substrain was provided by Dr. Leiselotte Herberg, Diabetes Research Institute, Düsseldorf, Germany) mice are a polygenic obese mouse model with elevated serum iron levels. Since adipose tissue remodeling is concomitant with high iron levels, this causes local adipose tissue insulin resistance [76]. db/db mice are an obesity model of lost function due to mutation in the gene encoding leptin receptor. It is characterized by obesity, insulin resistance, severe hyperglycemia, pancreatic injury, and cardiovascular complications, which support a model of metabolic disorder syndrome induced by obesity [77]. db/db mice present hippocampal neuroinflammation, which is consistent with obese chronic inflammation [78]. Stat5NKO mice are characterized by Stat5 locus deletion in the CNS, which triggers the development of severe hyperphagia-induced obesity, with impaired thermal regulation in response to cold, hyperleptinemia, and insulin resistance [79]. TRPV1 knockout (TRPV1-/-) mice exposed to high-fat food present more serious glucose intolerance, cardiac oxidative stress than normal mice [80].

Over ten years of acupuncture and EA research studies, obesity rodent models were the most widely used animal models. According to previous research studies, acupuncture in rodents showed positive outcomes, though these outcomes often differ from what has been described in humans. Among the rodent models used for acupuncture and EA studies, Sprague-Dawley rats and Wistar rats are the most represented (Table 2). Both strains have similar body size and acupoints localizations [89, 97, 113]. EA at Zusanli (ST36) in SD rats reduced bodyweight and inflammatory responses such as TNF- $\alpha$, IL-6, and IL- 1 levels in serum as well as mRNA expression in adipose tissue [90]. In the genetic Zucker rat obesity model, EA treatment at Zhongwan (RN12) and Guanyuan (RN4) acupoints led to significantly lower serum leptin and higher adiponectin/leptin ratio compared to an obese control group [114]. The decreased leptin may improve insulin sensitivity and increase insulin-stimulated glucose uptake in adipocytes [115]. Acupuncture at Zhongwan (CV12), Tianshu (ST25), Qihai (CV6), Ganshu (BL18), (Pishu) BL20, and Shenshu (BL23) significantly reduced blood glucose levels, with no effect on bodyweight [105]. EA at bilateral Zusanli (ST36) and Neiting (ST44) in Stat5NKO obesity mice reduced bodyweight, decreased plasma concentration of glucose, and reversed the altered gene expressions in the hypothalamus and epididymal white adipose tissue [109].

For some obesity rodent models, we found no example of acupuncture studies: this is the case for SHRSP/IDmcr-fas rats [71], db/db mice (loss of function mutation in the gene encoding leptin receptor) $[78,116], \mathrm{KK} / \mathrm{HlJ}$ mice [76], highfat diet induced obesity Swiss mice [64], and C57BL/6NCrl mice [65].

Traditional Chinese veterinary texts provide information on acupuncture points only for large animals. There is still no systematic acupuncture points' description in rodents. The situation differs in large animals such as pigs, sheep, horses, or dogs, for which veterinarians accumulated experience in using acupuncture or EA treatment. There are no veterinary medicine acupuncture textbooks describing acupoints on rats or mice. Most acupuncture or EA research studies using rodent models benefited from previously published studies for the acupoints localization $[85,110]$ or adapted the definition of rodent acupoints on the basis of the human anatomical acupoints location $[88,104]$. To date, we found no clear and systematic description of rodent acupoints in the general and scientific literature [96, 112], besides the fact that many acupoints cannot be identified in rodents because of significant anatomical differences compared to humans. Humans sleep and rest at night, which is consistent with the meridian work (circular motion) time dependent on the circadian rhythm, but rats and mice, as nocturnal species, normally exercised at night and sleep during daytime. In rodents, the small body size and thin muscle layer protecting the internal organs complicate acupuncture or EA because the risk for hurting pleural cavity or nerves in the leg is increased, which might lead to muscle atrophy. Unlike large animals, such as pigs [117], there is still no research about the description of rodent meridians, which makes more difficult and uncertain the implementation of acupuncture in these models. As a consequence, it is more difficult to interpret the outcomes of acupuncture or EA in rodents and to discuss the interindividual variability in terms of success or failure.

Rodents have a small and lissencephalic brain that makes brain explorations more difficult, technically speaking, and less relevant in terms of analogy with human. It is also more difficult to distinguish different muscles and nerves in rodents and to identify the precise spinal segments location for acupuncture points, especially in mice. This method is used for precise stimulation or inhibition of neuronal pathways in freely moving animals and is based on the transfection of a specific set of neurons with specific light sensitive proteins that can subsequently be activated by illumination $[118,119]$. In rodents, it is easier to perform repeated behavioral tests with many subjects than in large animals and nonhuman primates [120]. In the context of operant conditioning (lever pressing) with delayed rewards as executive function, obese Zucker rats learned to press the liver more quickly than lean animals. Their progressive ratio breakpoints (a measure of reward efficacy) 


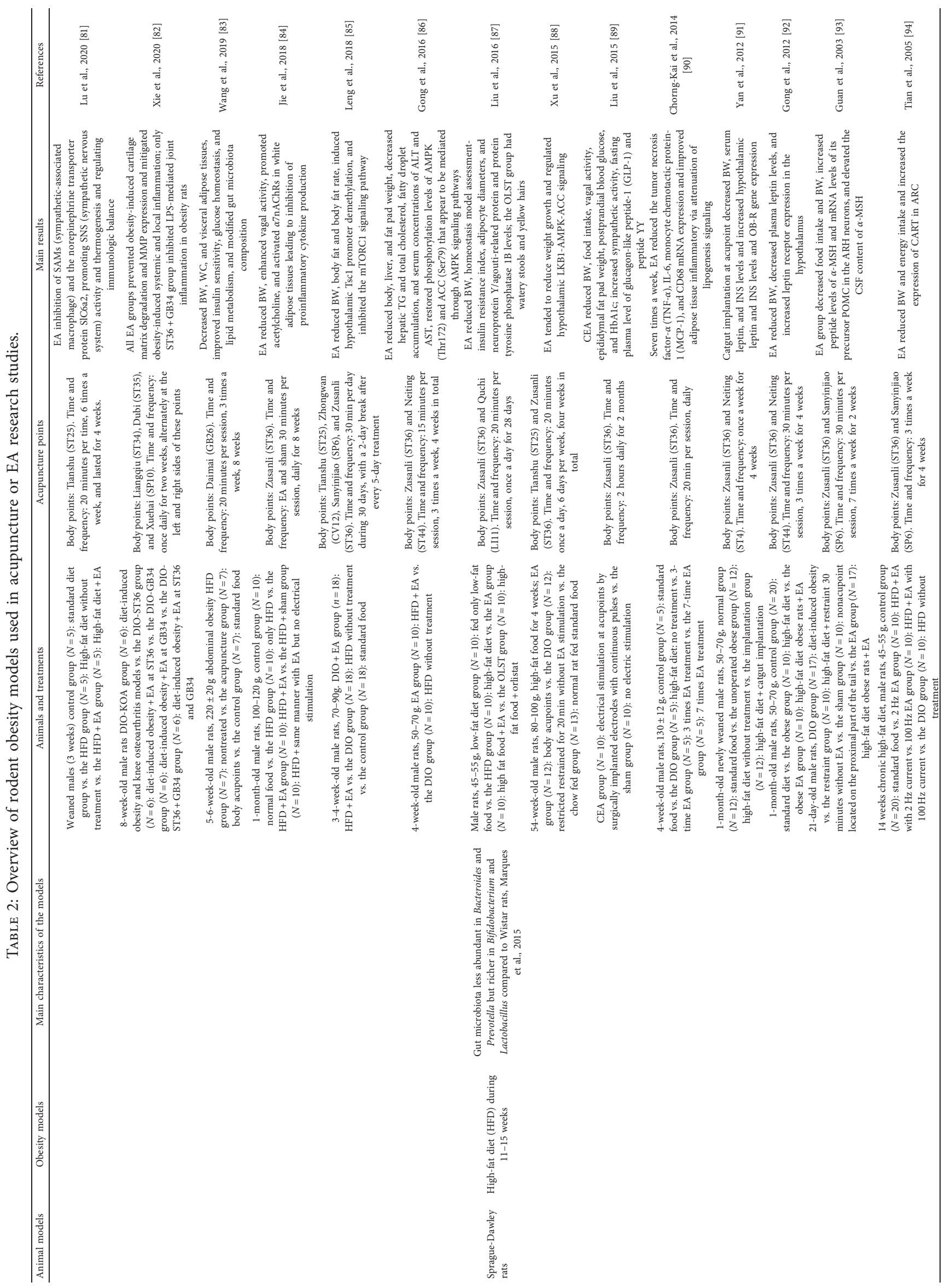




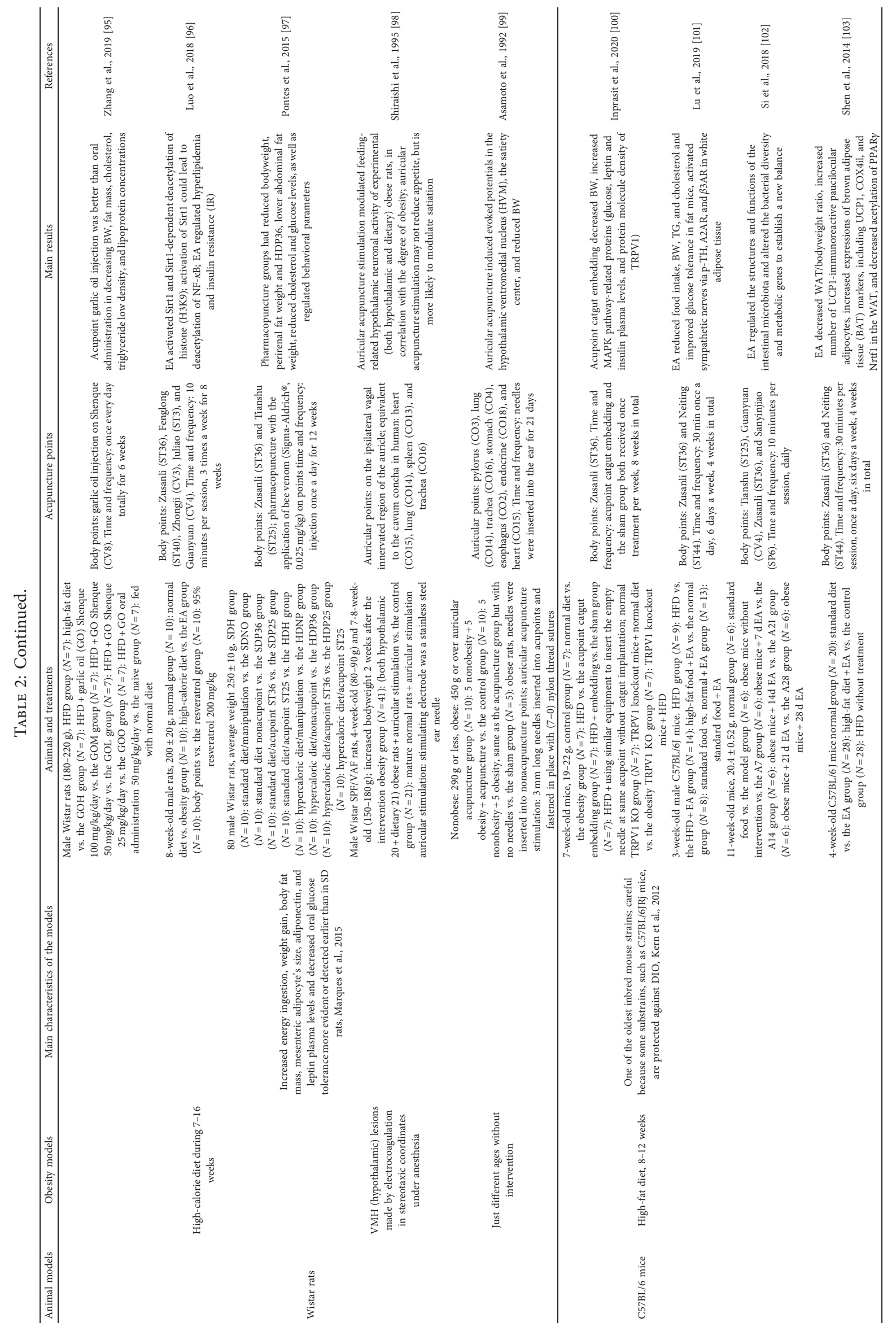




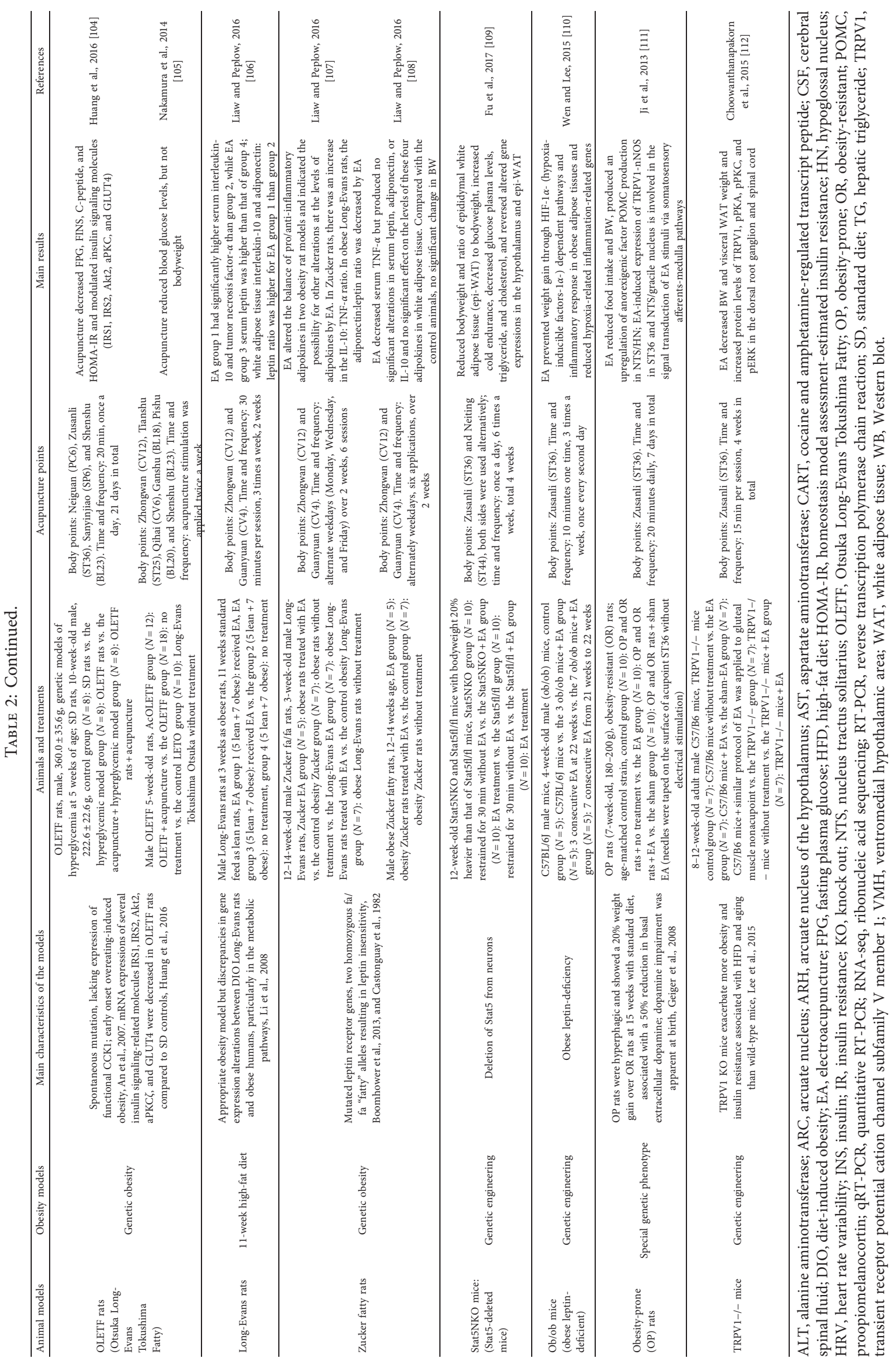


were as high as that of the lean group [121]. But in contrast with human, obese individuals were more likely to be present-focused, choosing smaller immediate rewards rather than larger delayed rewards (excessive delay discounting) $[122,123]$.

\section{Nonhuman Primate Models of Obesity and Acupuncture/Electroacupuncture}

Primates are the animals closest to humans in terms of phylogeny, metabolic physiology in the major sites of lipogenesis (adipose tissue and liver), and lipoprotein subclasses circulation, physiology of thermogenesis, and insulin-meditated glucose utilization [124]. Eight weeks of high-fat high-sugar diet is enough to increase fat mass and plasma triglycerides and reduce circulating adiponectin concentrations in baboons (Papio hamadryas) [125]. Feeding rhesus monkeys with fructose-sweetened solution daily during 6-12 months also induced many metabolic perturbations including central obesity and type- 2 diabetes [126]. High-fat maternal diet during pregnancy induced a dysbiosis in macaque's offspring, and these persistent alterations occurred despite cohousing of juvenile cohorts [127], which is coherent with results obtained in mice, where diet-induced maternal obesity can affect the progeny's gut microbiota, with persistent effects until young adulthood [128]. Like human without any diet restriction, free-ranging rhesus monkeys can also spontaneously develop obesity and diabetes on the island of Cayo Santiago $[129,130]$. Female cynomolgus macaques have been observed to develop spontaneous obesity in adulthood [131], with bodyweight showing positive correlation with increased serum leptin levels [132]. This trend is consistent with the correlation observed between the ob gene (which encodes leptin) expression and the percentage of body fat in humans [133]. With constant diet and environment, only part of the population progresses to diabetes, thus implicating genetic susceptibility factors that have not yet been described. Three novel single nucleotide polymorphisms (SNPs), two in apolipoprotein B (APOB) and one in phospholipase A2 (PLA2G4A), have been found associated with persistent weight stability and insulin sensitivity in lean macaques, which collectively produces an obesityresistant phenotype in adult female macaques [134]. A recent human meta-analysis of body mass index (BMI) genome-wide association studies estimated that 97 loci accounted for approximately $2.7 \%$ of BMI variation, and common variants accounted for up to $21 \%$ of BMI variation [135]. However, potentiating factors in human (i.e., diet, exercise, early life exposures, reproductive life-stage, sex, and comorbid metabolic conditions) make difficult to clearly define obese-susceptible and obese-resistant genomic variants.

Important similarities in the frontal cortex organization have been found in humans and other primates, even in the case of regions assumed to support human-specific functions. Areas in the human medial frontal cortex, including areas associated with high-level social cognitive processes such as theory of mind, showed a surprisingly high degree of similarity in their functional coupling patterns with the frontal pole, medial prefrontal, and dorsal prefrontal convexity in the macaque [136]. In capuchins and macaques, high-degree expansion was found in the temporal parietal junction, ventrolateral prefrontal cortex, and dorsal anterior cingulate cortex, all of which being involved in complex cognitive and behavioral functions. These expanded maps correlated well with previously published macaque-to-human registrations [137]. Between human and nonhuman primates, gray matter presents no significant difference, in relative terms, but the prefrontal white matter shows the largest differences and may have played an important role in human brain evolution [138]. From 358 dense individualized and common connectivity-based cortical landmarks (DICCCOL), 65 DICCCOLs are common in macaque monkey, chimpanzee, and human brains and demonstrated the consistencies of anatomical locations and structural fiber connection patterns between these species [139-141]. Furthermore, the monkey brain is overall structurally as variable as the human brain; low variability areas may have evolved less recently and have more stability, while high variability areas may have evolved more recently and be less similar across individuals [142].

Nonhuman primate models have been used in acupuncture research and provided interesting outcomes. The strong anatomical analogy with humans facilitates the localization of acupoints, which makes possible to identify more corresponding acupoints than in rodents, for example. In primate acupuncture research studies, the acupoints locations were referenced to acupuncture points anatomy locations in the human $[143,144]$. Chimpanzees were successfully trained to participate voluntarily in the acupuncture treatment, which avoids the use of anesthetics during acupuncture treatment and prevents any side effects of the anesthesia [143]. This also makes acupuncture more meaningful. Previous research using acupuncture treatment on Liangqiu (ST34), Dubi (ST35), and Zusanli (ST36) showed positive outcomes in chimpanzees with osteoarthritis and notably in two individuals with severe osteoarthritis of which the mobility was significantly improved [143]. Rhesus monkeys (Macaca mulatta) with Parkinsonian-like symptoms also received EA on Hegu (LI4) and Zusanli (ST36) after training without anesthesia and showed improved movement speed and muscle activity between arms after EA treatment [144]. According to high possibilities in terms of training of primates, complex behavioral and cognitive tests are possible and easier to perform [145]. Though, to date, we found no acupuncture or EA experiment reported on obese nonhuman primate models.

Amongst the main disadvantages of nonhuman primate models are their very long lifespan and related housing constraints, the very strict ethical regulations framing their use for research, as well as their susceptibility to transmit zoonotic diseases. Nonhuman primates are also very expensive due to specialized housing needs, the necessity for trained and knowledgeable husbandry and technical research staff, as well as the need for specific veterinary care. 


\section{Large Animal Models of Obesity and Acupuncture/Electroacupuncture}

Large animal models including pigs and sheep have been often used for obesity and nutrition research studies because they have more similar anatomical and physiological characteristics with humans compared to rodents. For example, 16-week high-fat diet exposure can make Dorset Horn greyface crossbreed sheep obese with metabolic disorders such as decreased blood-brain insulin transport, which contrasts with reports on genetically obese rats [146]. Among large animals, pigs and especially minipigs represent an economically and ethically promising substitute to nonhuman primates. Pigs have proportionally similar organ sizes and very comparable gastrointestinal tract anatomy, morphology, and physiology compared to humans and contrary to rodents [147]. Exposed to a high-fat diet, some minipig breeds easily develop weight gain and metabolic disorders, including highly specific brain anomalies comparable to those described in the human [148]. The Ossabaw swine is notably characterized with a "thrifty gene" phenotype propensity to store fat when exposed to excess calories [149]. Its increase in the transcript number and early transcriptomic alterations in the overall omental adipose tissue has been suggested to be a good model for studying childhood obesity [150].

Besides that, the pig brain has a convoluted or gyrencephalic cortical surface (Figure 1(b)), superficially resembling that of primates including humans $[151,152]$. The pig's skull is $40 \%$ thicker and the head twice as big compared to humans, which might sometimes produce artifact susceptibility during brain imaging, but strategies can be elaborated to optimize interspecies comparisons [153]. The pig's brain remains large enough to enable the identification of cortical and subcortical structures for neurosurgery and conventional imaging techniques in living animals. Pig brain is gyrencephalic and has a folded brain cortical surface with well-defined circumvolutions, an elongated oval shape with the hemispheres being widest at the posterior third and the occipital pole being larger than the frontal pole [154]. The olfactory system is more developed than in humans and occupies a large portion of the anterior part of the brain [151]. The brain cortex, including the somatosensory (SI) and prefrontal cortex (PFC) as well as their connections, the basal ganglia, and hypothalamus have been clearly described $[138,155]$. Recent anatomical research studies showed that the telencephalon of the Göttingen minipig cerebrum covers a large surface area, including neocortical gyrencephalic and ventral subrhinal parts, the first part being located dorsal to the rhinal fissure and the second part being dominated by olfactory, amygdaloid, septal, and hippocampal structures. The inner subcortical structure of the minipig telencephalon is dominated by a prominent ventricular system and large basal ganglia, wherein the putamen and the caudate nucleus posterior and dorsally are separated into two entities by the internal capsule, whereas both structures ventrally fuse into a large nucleus accumbens [156]. All these brain structures are primary regions of interest for the exploration of the brain correlates of food intake control. In comparison, the small lissencephalic brain of rodents makes more difficult functional imaging and translation to humans.

In the Göttingen minipigs, the prefrontal cortex activity was reduced after 5 months of ad libitum Western diet, which closely resembles brain anomalies previously described in obese humans [148]. Fed ad libitum during 41-47 months, Göttingen minipig also showed a deregulation of several obesity and inflammation-relevant protein-coding genes and miRNAs, of which many are also known to be deregulated in obese humans [157]. The minipig's offspring exposed to prenatal restricted nutrition [158] or maternal Western diet [159, 160] demonstrated obesity symptoms such as lower basal brain activity in the prefrontal cortex, cognitive and hedonic brain processes alterations, as well as negative changes in behavior and metabolism. All these studies demonstrate that minipig models are highly adapted to study the impact of obesity in the young or adult age, as well as the role of deleterious nutritional conditions during the perinatal period on further metabolic and behavioral imprinting.

Different pig models were created and used to investigate genetic factors related to obesity and its comorbidities. For example, a F2 pig generation was created upon a cross between lean production pigs (Yorkshire/Duroc) $\times$ Göttingen minipig before being extensively phenotyped for 36 obesity-related traits, revealing large phenotypic and genetic variations [161]. There are also genetically engineered pigs, such as transgenic miniature pigs expressing human ApoCIII, which were generated by the transfection of somatic cells combined with nuclear transfer. Transgenic pigs showed significantly higher triglyceride levels and delayed clearance of plasma triglycerides, accompanied by significantly reduced lipoprotein lipase activity in postheparin plasma [162]. Lp-phospholipase A2 (PLA2) transgenic swine has higher triglyceride levels and inflammatory gene, IL-6, MCP-1, and TNF- $\alpha$ mRNA in peripheral blood mononuclear cells. It was generated by eukaryotic expression plasmid and via somatic cell nuclear transfer. The overexpression of Lp-PLA2 was driven by EF1- $\alpha$ promoter [163].

In the context of behavioral tests, pigs usually have better performances than rats, for example, during motivation tests with a progressively increasing ratio between efforts and rewards [164]. Domestic pigs have the ability to discriminate between food sites of different relative values and to remember their respective locations. They are able to adjust their behavior when exposed to food sites with different food quantity and profitability [165]. Minipigs perform very well during cognitive tests such as the spatial hole-board discrimination task, the alley maze test, or two-choice food tests. In these paradigms, maternal Western diet piglets showed behavioral differences in comparison to maternal standard diet piglets, such as higher stress and lower performance in the alley maze [159]. It is consequently a good model to assess nutrition-related behavioral outcomes. In 


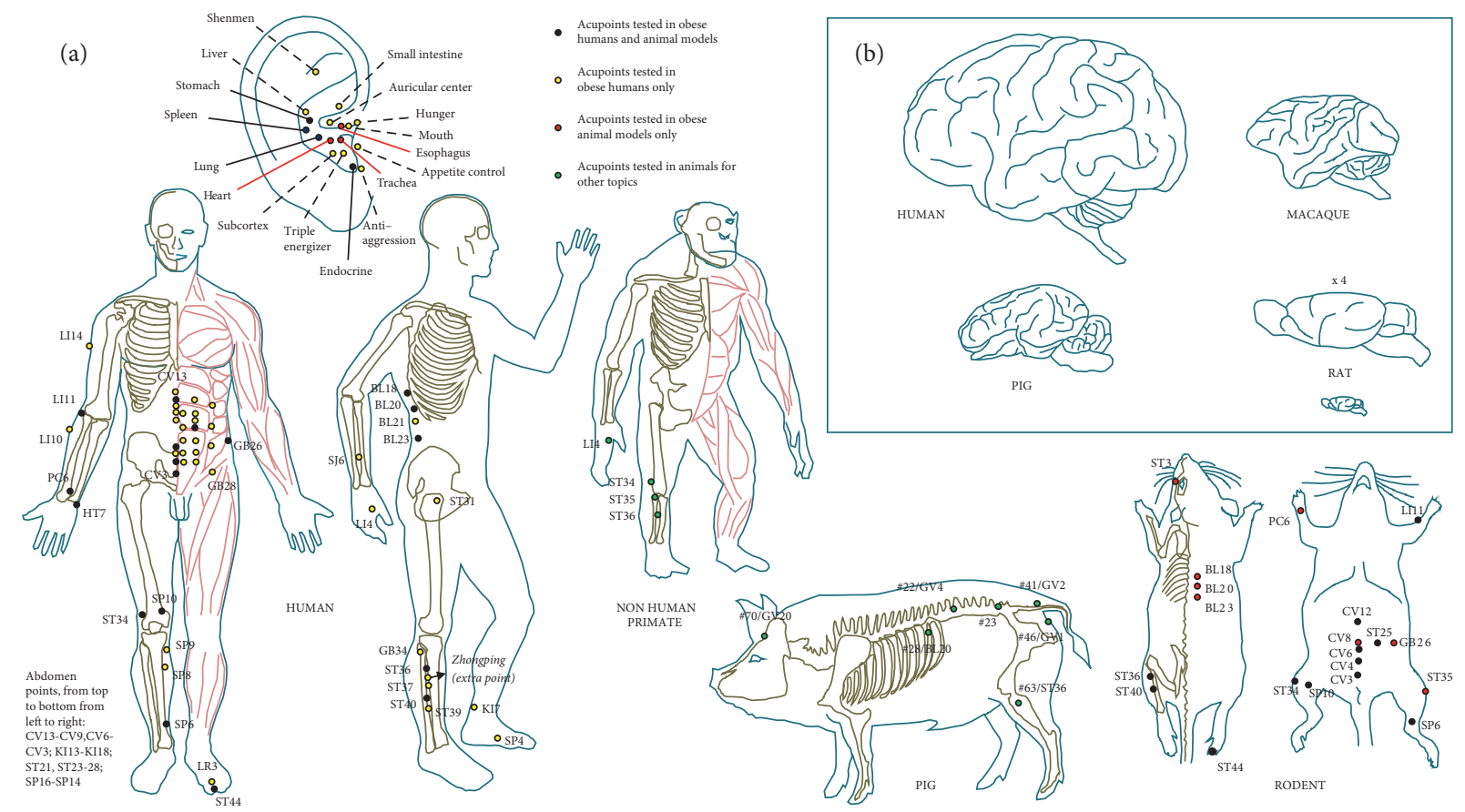

Figure 1: (a) Acupoints described and used in humans and different animal models including nonhuman primates, pigs, and rodents, mainly in the context of obesity and possibly in other topics. Auricular acupoints and body acupoints have been used in humans and animal models research studies. The codes for acupoints were indicated in accordance with the International Acupuncture Nomenclature proposed by the WHO. Pig acupoints were identified according to the textbook entitled "Traditional Chinese veterinary acupuncture and moxibustion" [12] and combined with human acupoints codes with comparable anatomical and structural landmarks. (b) Schematic representations of the brain in the human, macaque, pig, and rat.

OLETF rodents (a genetic obesity rat model characterized by spontaneous mutation, lacking expression of functional CCK1), lickometers were used to assess sugar preference, the interest in sucrose appearing only during early adulthood. The animals' preference for the higher sucrose concentrations was exacerbated along time and age [72]. The reason is still unknown, but the progressive maturation of the orosensory and hedonic systems, as well as the dopaminergic system mediating the reward value of sweet foods [166], might explain this difference in comparison to species with a quickest brain maturation, such as pigs. It has been demonstrated that juvenile pigs exposed to aversive or preferred foods present specific brain activity, especially in the reward circuit, demonstrating that they are perfectly able to discriminate the hedonic value of food [167]. Metabolic differences were notably observed in neural circuits known to be involved in humans in the characterization of food palatability, eating motivation, reward expectation, and more generally in the regulation of food intake [152]. In humans, exposure to flavors with different hedonic values induced eating motivation, reward expectation, and food intake regulation differences, all these processes being controlled by several brain structures including the amygdala and insular cortex [168]. Piglets also have higher acceptance of flavors that were present in the sow's diet during gestation and lactation [169], which is consistent with what has been described in human babies [170].

The precise description of acupoints in pigs, equine, bovine, porcine, sheep, camel, canine, and rabbit can be found in the textbook entitled traditional Chinese veterinary acupuncture and moxibustion [12], which has accumulated many years of veterinarians' clinical experience. Large animals such as pigs, cows, and dogs have good reactions to acupuncture, with positive health outcomes. In veterinary medicine, acupuncture has been widely used to treat swine diseases [171]. For instance, Changqiang (GV1), Yaoshu (GV2), Mingmen (GV4), and Baihui (GV20) are the usual choice to treat impotence and penile paralysis in male boars [172]. In the early stage of inoculation with Escherichia coli, acupuncture treatment on Changqiang (GV1), Baihui (GV20), Pishu (BL20), and Zusanli (ST36) had a better effect than neomycin on diarrhea and gut inflammation in young pigs [173]. A twoweek treatment on Dafengmen (\#70), of which the anatomical location is similar to human Baihui (GV20), significantly improved sleep quality in pigs and changed the catecholamine levels in pooled urine [174]. Tianzhu (BL10) and Dazhu (BL11), as well as Fengchi (GB20) and Jianjing (GB21) have a good effect on paralytic or lame dogs [172]. Hemoacupuncture (a technique encouraging a small amount of bleeding from specific acupuncture points) at Shaoze (SI1), Guanchong (SJ1), Shangyang (LI1), and Qiantitou, as well as acupuncture on Shaoshang (LU11), Shaochong (HT9), Zhongchong (PC9), and Qiantimen, had positive effects on horse with chronic laminitis [175]. Twelve weeks of acupuncture at Baihui (GV20) and Ashi points in mildly lame horse improved horses' gaits to an appreciable degree by objective and subjective analyses [14]. The injection of $125 \mu \mathrm{g} /$ $\mathrm{m}^{2}$ dexmedetomidine in acupuncture point Baihui (GV20) in 
dogs increased the duration and degree of sedation and analgesic effects of dexmedetomidine compared with intramuscular injected in loci that did not correspond to acupuncture points [176]. After 24 weeks of treatment, acupuncture alone and combined with analgesics both improved Helsinki chronic pain index (HCPI), visual analog scales (VAS), and improved life quality in 181 dogs with neurological and musculoskeletal diseases [177].

Because the blocking of low hydraulic resistance channel of the minipig stomach meridian by gel triggered gastric and intestinal distension, it has been suggested that minipigs have a meridian reactivity similar to humans. Indeed, according to basic Chinese medicine theory, human stomach meridian controls digestion and intestinal stomach movement [117]. In order to find in animals the best analogy with human acupuncture points, it is important to rely on anatomical landmarks and not only on the names given to the acupoints in the animal. For example, the human Baihui (GV20) acupoint is located on the midline of the head vertex. In pig, there is a so-called "Baihui" acupoint, but it is located at the top of the back near the hip and has nothing to do with human Baihui. The pig acupoint \#70 Dafengmen has an anatomical location similar to human Baihui, and as in humans, its stimulation can modulate urine catecholamine and improve sleep condition [174].

Surprisingly, we found no study aimed at investigating the effects of acupuncture or EA in a pig model of obesity or hyperphagia (an abnormally strong sensation of hunger or desire to eat often leading to or accompanied by overeating) nor in any other large animal model, which highlights the complete lack or research in this promising field. We recently published a hypothesis study [178] presenting the rationale and a methodological approach to investigate the effects of electroacupuncture in obese minipigs. This ongoing study is the first of its kind. The acupuncture points that were selected for this work included three combinations of paired acupoints, i.e., Pishu (\#28) and Liumai (\#27) on the back, Dafengmen (\#70) on the head, and Sanwan (\#35) on the abdomen, as well as Hangou (\#79) and Housanli (\#63) on the hind legs. Our preliminary results obtained in normal weight animals showed that acute EA did not modify the glucose and insulin plasma levels, but had a tendency to modulate the heart rate variability (HRV) assessed via electrocardiography (ECG), which is a proxy for vagus tone. Most interesting is the fact that the combination Dafengmen/Sanwan produced significant BOLD fMRI activation of the prefrontal cortex, striatum, hippocampus, and cingulate cortex, in comparison to the other combinations or a sham treatment (Zhang and Val-Laillet, in preparation). This combination is currently being tested in obese Yucatan minipigs in the context of a one-month chronic treatment with 3 EA sessions per week.

\section{General Discussion and Conclusions}

6.1. About the Heterogeneity of Human Clinical Trials: A Rational for Preclinical Studies. Acupuncture and EA are being increasingly popular in clinical and preclinical research. Because of the ethical, financial, and practical constraints associated with randomized controlled trials (RCT), not all research on acupuncture and related therapies can be performed in the human. Preclinical explorations are needed before the onset of RCT, and the use of relevant animal models is consequently justified. Animal studies also have the advantage to better control factors that are extremely variable and difficult to assess in human populations. Human subjects usually present diverse degrees of cooperation or understanding during a clinical trial, and it is often difficult to survey precisely their food intake and physical activity. A recent review highlighted the relative ambiguities regarding the role of physical activity in obesity treatment, and its impact on body composition sometimes shows some inconsistencies $[179,180]$. Many clinical studies fail to adequately measure or assess calorie intake, concurrent changes in habitual activity, non-prescribed weightloss behavioral strategies, or inconsistent dose (duration and intensity) of physical exercise across intervention types. Incomplete data collection further contributes to the methodological heterogeneity between clinical trials. The question of sham and real acupuncture still has not reached consistent conclusions either. Some authors show that sham stimulations (noninsertive acupuncture or nonacupuncture points) do not differ from real acupuncture [181], while others, for example, demonstrated in obese women some positive effects of acupuncture and ear point seeded compared to sham treatment on fasting insulin, HOMA-IR, and inflammation factors [27]. Further research is needed on this topic, and the question of sham acupuncture is probably much easier to treat in animal models than in humans who are subjected to psychosomatic factors and other cognitive biases towards the outcomes of a medical treatment. As highlighted in Table 1, various types of sham treatments have been used in the human, which complicates the comparison between studies.

Similarly, the multiplicity of disease models and combinations of acupoints selected makes very difficult a systematic comparison of the outcomes, which can significantly differ between studies and species. There is still no consensus guidance to select acupuncture points for obesity treatment, and many questions about the underlying physiological and behavioral mechanisms of these effects remain unanswered. From previous research studies, different acupuncture points can produce different effects. As obesity derives from complex metabolic and neurobehavioral processes including many parameters and potentially generating diverse pathological profiles, further research is needed to describe precise candidate acupoint targets and their related effects in terms of physiology and behavior. To investigate pathological and gene mechanisms, tissue sampling is often mandatory, which is difficult to perform in humans but very easy in animal models. Several authors highlighted the important role of the central nervous system and the communication pathways though it is possible to leverage brain processes in the effects of acupuncture or EA stimulation on diseases [182]. Such research usually requires histological and molecular biology studies to understand the complex mechanisms underlying these outcomes. 
6.2. Interspecies Comparison about Ear Acupuncture and Related Therapies. From acupuncture research in humans (Table 1), we found that ear acupuncture points (Figure 1(a)) have been widely investigated in clinical trials, with positive outcomes in obese humans in terms of appetite, bodyweight and BMI, plasma lipidemia, as well as ghrelin and leptin levels, for example $[22,31,34,39,47]$. The reasons for these outcomes are still unknown but are probably related to complex neurophysiological mechanisms. In the human, the cymba conchae stimulation in the external ear by continuous wave significantly activates the classical central vagal projections (widespread activity in the ipsilateral NTS, bilateral spinal trigeminal nucleus, dorsal raphe, locus coeruleus, and contralateral parabrachial area, amygdala, and nucleus accumbens) [183]. All these brain regions are involved in homeostatic and hedonic food intake control. In rats, stimulation at auricular points pylorus (CO3), lung (CO14), trachea (CO16), stomach (CO4), esophagus (CO2), endocrine $(\mathrm{CO} 18)$, and heart $(\mathrm{CO} 15)$ has been reported to activate the hypothalamic ventromedial nucleus satiety center and reduce bodyweight [99]. Other research studies related to auricular acupuncture in obesity animal models are very scarce because rats and mice have very small ears. Mice are characterized by "microtype" ears with microtype mallei associated with high-frequency hearing which is not found in humans [184]. Though, some authors demonstrated in obese rats, for example, that auricular acupuncture stimulation reduced bodyweight [99], modulated feeding-related hypothalamic neuronal activity [98], reduced epididymal WAT, and increased brown adipose tissue (BAT) weight, serum norepinephrine, mRNA expressions of $\beta 3$-adrenoceptors, and UCP1 of the BAT [185]. Compared to rodents, pigs have large ears, of which the general anatomy is more likely comparable to those in humans. Also, the obtained transfer function is congruent to human [186]. Though, we found only one study using ear acupoints in combination with other body acupoints in this species, with beneficial effects in controlling Escherichia coli diarrhea [173]. Most nonanthropoids primates have tall and narrow ears, the ears of monkeys and apes being more equal in terms of height and width [187].

\subsection{Bridging the Gap between Classical Animal Models and} Humans in EA Research. As highlighted in Table 2, we found that acupuncture or EA research studies in rodents were limited to several acupuncture points, especially Zusanli (ST36), because the significant differences with humans in terms of body surface/volume and anatomical structures make difficult to find more analogies with the acupuncture points in humans (Figure 1(a)). In rodent obesity models, acupuncture or EA also work well on anthropometric index and biological indicators (Table 2). But first, some rodent models, especially genetically engineered rodents, cannot explain modern human epidemic obesity with complex triggering factors (diet habits, environmental pressures, and genes), therefore, limiting their construct validity. Second, some RNA or specific protein changes after EA or acupuncture in obesity have not been confirmed in humans, and the visible changes that can directly explain acupuncture or EA effects on brain reactivity, for example, are still very rare in rodent models because of methodological and resolution restrictions, which limit their face validity. Third, some acupuncture strategies that were successful in reducing bodyweight in the human [39] did not do so in rats [104, 105], therefore, limiting the predictive validity of this model.

Genes may play an important role in the onset of obesity, and converging data were obtained in several species. The OLETF rat, with spontaneous mutation characterized by lacking the expression of functional CCK1, is an early onset overeating-induced obesity model [72]. Though, there is still no evidence of the existence of a CCK1 mutation in dietinduced obesity human, which limits the extrapolation of data from this rat model. In macaques, three novel SNPs have been found associated with obesity-resistant phenotype [134]. In humans, 22 obesity-related candidate SNP markers have been found through DNA sequence analysis by using web service SNP_TATA_Comparator and keyword articles search [99]. In rodents, in intersubspecific backcross population between C57BL/6JJcl (B6) and wild Mus musculus castaneus mice, Gcg and Grb14, Ly75, and Itgb6 were suggested candidate genes, but these genes had nonsynonymous SNPs [188]. Genetically engineered models can produce different results for the same parameters. For example, in genetically Zucker obese rats, blood-brain insulin transport is decreased [189], which contrasts with data in large animals. For example, decreased proportional bloodbrain insulin transport was associated with weight loss in obese sheep, but the central insulin resistance (in terms of food intake response) resulted from intrahypothalamic insensitivity rather than impaired blood-brain insulin transfer [146].

Undoubtedly, nonhuman primate obesity models are closer to humans and allow for better analogy when investigating the effects of acupuncture or EA, but the higher cost and ethical restrictions limit their use. Rodent models also have advantages, since they are easily bred, housed, and handled at lower costs and with less ethical restrictions than larger animals. It is also very easy to produce genetically engineered obesity models. It should be noted also that small animal models such as mice can represent an asset for certain techniques, such as optogenetics, in order to investigate the effect of acupuncture or EA regulation on the neural basis of pathological behavior, for example [190]. Rodents and especially mice have a thinner skull compared to other large animals and are easier to handle. The recent development of optogenetics permits real-time manipulation of region and cell type-specific neural pathways in awake behaving rodents [191, 192]. Optogenetics has been used to reversibly control depression-related phenotypes in mice by manipulating the medial prefrontal cortex (mPFC) [193], for example, and further implementation of this technique in the scope of EA research should be investigated.

To date, obesity animal models for acupuncture and related therapies are restricted to rodents, which significantly limits the extrapolation and translation to human. It is, therefore, necessary to propose alternative preclinical strategies, benefitting from innovative obese animal models 
to support further acupuncture research studies. Large animal obesity models appear like a bridge between rodents and nonhuman primates. Pigs, especially minipigs, are closer to humans than rodents, less expensive, and difficult to breed than nonhuman primates. This species also has been extensively used for nutritional and neurosciences studies, making possible crossed obesity and brain research combined with complex behavioral explorations [147, 151, 152]. They also are very interesting for behavioral studies aimed at investigating the different dimensions of eating behavior, including individual preferences and choices, motivation, and the classical food liking and wanting usually explored in human subjects [194]. In humans, overeating usually emerges when food signals sensitivity and appetitive motivation to consume palatable foods overrule the inhibitory control of eating [195]. Comparable data have been obtained in the adult minipig model $[148,196]$, as well as in the context of the developmental origins of health and diseases $[159,160,197]$, in terms of behavior and brain functioning.

On the specific question of brain reactivity to acupuncture or EA, research studies are rare and almost restricted to human research $[52,53]$. The mechanisms and effects of different acupuncture points are still poorly described. The small lissencephalic brain in rodents (Figure 1(b)) significantly restricts neurobiological and neuroimaging research studies, and such studies in the human are costly and limited by practical and ethical constraints. Animal models with brain anatomical structures and functions more similar to those in humans are necessary, and the pig has a brain very close to that of macaques, for example, with many circumvolutions as in humans (Figure 1(b)). The fact that pig brain anomalies in the context of disordered eating and obesity are very similar to those described in the human is another potent argument to support the use of this model to investigate the neurobiological mechanisms of acupuncture and related therapies.

In the spectrum of obesity treatments, obesity surgery is usually reserved for severe obesity or moderate obesity accompanied with significant comorbidities. It is often the last recourse after failure of other treatments, but it is frequently associated with nutritional risks and potential complications. In addition or complement to obesity surgery, antiobesity medicine is one of the main treatments, but most FDA-approved antiobesity drugs have significant individual variations in response rates [198]. Long-term safety, potential adverse effects, and relative efficacy also are some of the main reasons why very few patients accept them. Most of these medicines can provide greater weight loss than do lifestyle changes alone, although few promising antiobesity medications are in the drug-development pipeline. The most promising drugs are novel molecules that are coagonists for multiple gut hormones including GLP-1, glucagon, and gastric inhibitory peptide [200]. Contrary to pharmacological treatments, acupuncture and related therapies have very few adverse effects, such as nausea, mild and transient syncope, and very rare events of septicaemia and hepatitis $\mathrm{C}$ infection [200]. Most of these rare events can be avoided with the use of disposable needles and good disinfection and sanitary conditions. Acupuncture alone or in combination with other therapies gains increasing attention, but the underlying mechanisms and the best acupoint combinations are still unknown. Further research studies with proper obesity animal models are needed to find the most effective treatment parameters and decipher their underlying mechanisms.

\section{Conflicts of Interest}

Xuwen Zhang received a grant from the International Program for Postgraduates, Guangzhou University of Chinese Medicine. The authors declare that they have no conflicts of interest.

\section{References}

[1] WHO, Obesity and Overweight, http://www.who.int/ mediacentre/factsheets/fs311/en/.

[2] A. Elagizi, S. Kachur, C. J. Lavie et al., "An overview and update on obesity and the obesity paradox in cardiovascular diseases," Progress in Cardiovascular Diseases, vol. 61, no. 2, pp. 142-150, 2018.

[3] Y. Liu and Z. Ding, "Obesity, a serious etiologic factor for male subfertility in modern society," Reproduction, vol. 154, no. 4, pp. R123-R131, 2017.

[4] J. N. Farr and P. Dimitri, "The impact of fat and obesity on bone microarchitecture and strength in children," Calcified Tissue International, vol. 100, no. 5, pp. 500-513, 2017.

[5] D. S. Michaud, E. Giovannucci, W. C. Willett, G. A. Colditz, M. J. Stampfer, and C. S. Fuchs, "Physical activity, obesity, height, and the risk of pancreatic cancer," Journal of the American Medical Association, vol. 286, no. 8, pp. 921-929, 2001.

[6] M. R. Fernandes, N. V. d. Lima, K. S. Rezende, I. C. M. Santos, I. S. Silva, and R. d. C. A. Guimarães, "Animal models of obesity in rodents. An integrative review," Acta Cirurgica Brasileira, vol. 31, no. 12, pp. 840-844, 2016.

[7] M. Kleinert, C. Clemmensen, S. M. Hofmann et al., "Animal models of obesity and diabetes mellitus," Nature Reviews Endocrinology, vol. 14, no. 3, pp. 140-162, 2018.

[8] M. J. Den Broeder, V. A. Kopylova, L. M. Kamminga, and J. Legler, "Zebrafish as a model to study the role of peroxisome proliferating-activated receptors in adipogenesis and obesity," PPAR Research, vol. 2015, Article ID 358029, 11 pages, 2015.

[9] A. Teleman, I. Ratzenböck, and S. Oldham, "Drosophila: a model for understanding obesity and diabetic complications," Experimental and Clinical Endocrinology \& Diabetes, vol. 120, no. 4, pp. 184-185, 2012.

[10] T. A. Lutz and S. C. Woods, "Overview of animal models of obesity," Current Protocols in Pharmacology, vol. 5, 2013.

[11] R. Guo, "Biomedical acupuncture for pain management," Focus on Alternative \& Complementary Therapies, vol. 11, no. 4, p. 344, 2007.

[12] C. Yu, Traditional Chinese Veterinary Acupuncture and Moxibustion, China Agriculture Press, Beijing, China, 1995.

[13] W.-W. Chan, K.-Y. Chen, H. Liu, L.-S. Wu, and J.-H. Lin, "Acupuncture for general veterinary practice," Journal of Veterinary Medical Science, vol. 63, no. 10, pp. 1057-1062, 2001.

[14] B. Dunkel, T. Pfau, A. Fiske-Jackson et al., "A pilot study of the effects of acupuncture treatment on objective and 
subjective gait parameters in horses," Veterinary Anaesthesia and Analgesia, vol. 44, no. 1, pp. 154-162, 2017.

[15] C. M. Apovian, "Obesity: definition, comorbidities, causes, and burden," American Journal of Managed Care, vol. 22, no. 7 Suppl, pp. s176-s185, 2016.

[16] G. Seravalle and G. Grassi, "Obesity and hypertension," Pharmacological Research, vol. 122, pp. 1-7, 2017.

[17] Y. Zhang, J. Li, G. Mo et al., "Acupuncture and related therapies for obesity: a network meta-analysis," EvidenceBased Complementary and Alternative Medicine, vol. 2018, Article ID 9569685, 20 pages, 2018.

[18] V. M. Jackson, D. M. Breen, J.-P. Fortin et al., "Latest approaches for the treatment of obesity," Expert Opinion on Drug Discovery, vol. 10, no. 8, pp. 825-839, 2015.

[19] P. J. Nathan, B. V. O’Neill, A. Napolitano, and E. T. Bullmore, "Neuropsychiatric adverse effects of centrally acting antiobesity drugs," CNS Neuroscience and Therapeutics, vol. 17, no. 5, pp. 490-505, 2011.

[20] K. W. Kim, W. C. Shin, M. S. Choi et al., "Effects of acupuncture on anthropometric and serum metabolic parameters in premenopausal overweight and obese women: a randomized, patient- and assessor-blind, sham-controlled clinical trial," Acupuncture in Medicine, vol. 39, no. 1, pp. 30-40, 2021.

[21] F. Lillingston, P. Fields, and R. Waechter, "Auricular acupuncture associated with reduced waist circumference in overweight women-A randomized controlled trial," Evidence-Based Complementary and Alternative Medicine, vol. 2019, Article ID 6471560, 7 pages, 2019.

[22] H. S. Cha and H. Park, "Effects of auricular acupressure on obesity in adolescents," Complementary Therapies in Clinical Practice, vol. 35, pp. 316-322, 2019.

[23] X. Zhou, B. Xing, G. He, X. Lyu, and Y. Zeng, "The effects of electrical acupuncture and essential amino acid supplementation on sarcopenic obesity in male older adults: a randomized control study," Obesity Facts, vol. 11, no. 4, pp. 327-334, 2018.

[24] H. Lei, X. Chen, S. Liu, and Z. Chen, "Effect of electroacupuncture on visceral and hepatic fat in women with abdominal obesity: a randomized controlled study based on magnetic resonance imaging," Journal of Alternative \& Complementary Medicine, vol. 23, no. 4, pp. 285-294, 2017.

[25] M. Mazidi, P. Abbasi-Parizad, and H. Abdi, "The effect of electro-acupuncture on pro-oxidant antioxidant balance values in overweight and obese subjects: a randomized controlled trial study," Journal of Complementary and Integrative Medicine, vol. 15, no. 2, 2018.

[26] C. Yasemin, S. Turan, and Z. Kosan, "The effects of auricular and body acupuncture in Turkish obese female patients: a randomized controlled trial indicated both methods lost body weight but auricular acupuncture was better than body acupuncture," Acupuncture \& Electro-Therapeutics Research, vol. 42, no. 1, pp. 1-10, 2017.

[27] A. Firouzjaei, G.-C. Li, N. Wang, W.-X. Liu, and B.-M. Zhu, "Comparative evaluation of the therapeutic effect of metformin monotherapy with metformin and acupuncture combined therapy on weight loss and insulin sensitivity in diabetic patients," Nutrition \& Diabetes, vol. 6, no. 5, p. e209, 2016.

[28] M. Belivani, T. Lundeberg, M. Cummings et al., "Immediate effect of three different electroacupuncture protocols on fasting blood glucose in obese patients: a pilot study," Acupuncture in Medicine, vol. 33, no. 2, pp. 110-114, 2015.
[29] J. He, X. Zhang, Y. Qu et al., "Effect of combined manual acupuncture and massage on body weight and body mass index reduction in obese and overweight women: a randomized, short-term clinical trial," Journal of Acupuncture and Meridian Studies, vol. 8, no. 2, pp. 61-65, 2015.

[30] L. A. Abou Ismail, A. A. Ibrahim, G. A. Abdel-Latif et al., "Effect of acupuncture on body weight reduction and inflammatory mediators in Egyptian obese patients," Open Access Macedonian Journal of Medical Sciences, vol. 3, no. 1, pp. 85-90, 2015.

[31] M.-L. Yeh, N.-F. Chu, M.-Y. F. Hsu, C.-C. Hsu, and Y.-C. Chung, "Acupoint stimulation on weight reduction for obesity," Western Journal of Nursing Research, vol. 37, no. 12, pp. 1517-1530, 2015.

[32] S. Fogarty, L. Stojanovska, D. Harris, C. Zaslawski, M. L. Mathai, and A. J. McAinch, "A randomised cross-over pilot study investigating the use of acupuncture to promote weight loss and mental health in overweight and obese individuals participating in a weight loss program," Eating and Weight Disorders-Studies on Anorexia, Bulimia and Obesity, vol. 20, no. 3, pp. 379-387, 2015.

[33] J. Wu, Q. Li, L. Chen, and D. Tian, "Clinical research on using acupuncture to treat female adult abdominal obesity with spleen deficiency and exuberant dampness," Journal of Traditional Chinese Medicine, vol. 34, no. 3, pp. 274-278, 2014.

[34] M. Darbandi, S. Darbandi, A. A. Owji et al., "Auricular or body acupuncture: which one is more effective in reducing abdominal fat mass in Iranian men with obesity: a randomized clinical trial," Journal of Diabetes and Metabolic Disorders, vol. 13, no. 1, p. 92, 2014.

[35] D. Kim, O. K. Ham, C. Kang, and E. Jun, "Effects of auricular acupressure using Sinapsis alba seeds on obesity and selfefficacy in female college students," Journal of Alternative \& Complementary Medicine, vol. 20, no. 4, pp. 258-264, 2014.

[36] S. Yeo, K. S. Kim, and S. Lim, "Randomised clinical trial of five ear acupuncture points for the treatment of overweight people," Acupuncture in Medicine, vol. 32, no. 2, pp. 132-138, 2014.

[37] R. P. Schukro, C. Heiserer, A. Michalek-Sauberer, A. Gleiss, and S. Sator-Katzenschlager, "The effects of auricular electroacupuncture on obesity in female patients - a prospective randomized placebo-controlled pilot study," Complementary Therapies in Medicine, vol. 22, no. 1, pp. 21-25, 2014.

[38] H. Abdi, B. Zhao, and M. Darbandi, "The effects of body acupuncture on obesity: anthropometric parameters, lipid profile, and inflammatory and immunologic markers," ScientificWorldJournal, vol. 2012, Article ID 603539, 11 pages, 2012.

[39] F. Güçel, B. Bahar, C. Demirtas, S. Mit, and C. Çevik, "Influence of acupuncture on leptin, ghrelin, insulin and cholecystokinin in obese women: a randomised, shamcontrolled preliminary trial," Acupuncture in Medicine, vol. 30, no. 3, pp. 203-207, 2012.

[40] C. H. Hsieh, T. J. Su, Y. W. Fang, and P. H. Chou, "Effects of auricular acupressure on weight reduction and abdominal obesity in Asian young adults: a randomized controlled trial," American Journal of Chinese Medicine, vol. 39, no. 3, pp. 433-440, 2011.

[41] C. H. Hsieh, "The effects of auricular acupressure on weight loss and serum lipid levels in overweight adolescents," American Journal of Chinese Medicine, vol. 38, no. 4, pp. 675-682, 2010. 
[42] C. H. Lin, Y. M. Lin, and C. F. Liu, "Electrical acupoint stimulation changes body composition and the meridian systems in postmenopausal women with obesity," American Journal of Chinese Medicine, vol. 38, no. 4, pp. 683-694, 2010.

[43] C.-H. Hsu, C.-J. Wang, K.-C. Hwang, T.-Y. Lee, P. Chou, and H.-H. Chang, "The effect of auricular acupuncture in obese women: a randomized controlled trial," Journal of Women's Health, vol. 18, no. 6, pp. 813-818, 2009.

[44] C.-H. Hsu, K.-C. Hwang, C.-L. Chao, H.-H. Chang, and P. Chou, "Electroacupuncture in obese women: a randomized, controlled pilot study," Journal of Women's Health, vol. 14, no. 5, pp. 434-440, 2005.

[45] C.-H. Hsu, K.-C. Hwang, C.-L. Chao, J.-G. Lin, S.-T. Kao, and P. Chou, "Effects of electroacupuncture in reducing weight and waist circumference in obese women: a randomized crossover trial," International Journal of Obesity, vol. 29, no. 11, pp. 1379-1384, 2005.

[46] R. G. Sebayang, C. Aditya, K. Abdurrohim et al., "Effects of laser acupuncture and dietary intervention on key obesity parameters," Medical Acupuncture, vol. 32, no. 2, pp. 108-115, 2020.

[47] C. C. Tseng, A. Tseng, J. Tseng, and C. H. Chang, "Effect of laser acupuncture on anthropometric measurements and appetite sensations in obese subjects," Evidence-Based Complementary and Alternative Medicine, vol. 2016, Article ID 9365326, 8 pages, 2016.

[48] L.-S. Chen, Y.-Y. Li, H. Chen, B.-W. Liu, D.-W. Wang, and Y.-H. Zhao, "Polyglycolic acid sutures embedded in abdominal acupoints for treatment of simple obesity in adults: a randomized control trial," Chinese Medicine, vol. 14, no. 1, p. 32, 2019.

[49] J. M. Garcia-Vivas, C. Galaviz-Hernandez, J. FernandezRetana et al., "Transcriptomic profiling of adipose tissue in obese women in response to acupuncture catgut embedding therapy with moxibustion," Journal of Alternative \& Complementary Medicine, vol. 22, no. 8, pp. 658-668, 2016.

[50] Y.-C. Hung, I.-L. Hung, W.-L. Hu et al., "Reduction in postpartum weight with laser acupuncture," Medicine (Baltimore), vol. 95, no. 34, Article ID e4716, 2016.

[51] J. M. Garcia-Vivas, C. Galaviz-Hernandez, F. BecerrilChavez et al., "Acupoint catgut embedding therapy with moxibustion reduces the risk of diabetes in obese women," Journal of Research in Medical Sciences, vol. 19, no. 7, pp. 610-616, 2014.

[52] Y. Ren, M. Xu, K. M. von Deneen et al., "Acute and longterm effects of electroacupuncture alter frontal and insular cortex activity and functional connectivity during resting state," Psychiatry Research: Neuroimaging, vol. 298, Article ID 111047, 2020.

[53] K. M. von Deneen, W. Qin, and P. Liu, "Connectivity study of the neuromechanism of acute acupuncture needling during fMRI in "overweight" subjects," Evidence-Based Complementary and Alternative Medicine, vol. 2015, Article ID 384389, 12 pages, 2015.

[54] S. K. Avants, T. R. Margolin A Fau-Holford, T. R. Holford Tr Fau-Kosten, and T. R. Kosten, "A randomized controlled trial of auricular acupuncture for cocaine dependence," Archives of Internal Medicine, vol. 160, no. 15, pp. 23052320, 2001.

[55] M. L. Bullock, R. T. Culliton, and R. T. Olander, "Controlled trial of acupuncture for severe recidivist alcoholism," Lancet, vol. 1, no. 8652, pp. 1435-1439, 1989.

[56] M. Shwartz, R. Saitz, K. Mulvey, and P. Brannigan, "The value of acupuncture detoxification programs in a substance abuse treatment system," Journal of Substance Abuse Treatment, vol. 17, no. 4, pp. 305-312, 1999.

[57] J. A. Chen, J. A. Chen, S. Lee, and G. Mullin, "Potential role for acupuncture in the treatment of food addiction and obesity," Acupuncture in Medicine, vol. 36, no. 1, pp. 52-55, 2018.

[58] A. Constant, R. Moirand, R. Thibault, and D. Val-Laillet, "Meeting of minds around food addiction: insights from addiction medicine, nutrition, psychology, and neurosciences," Nutrients, vol. 12, no. 11, p. 3564, 2020.

[59] K. Zhang, S. Zhou, C. Wang, H. Xu, and L. Zhang, "Acupuncture on obesity: clinical evidence and possible neuroendocrine mechanisms," Evidence-Based Complementary and Alternative Medicine, vol. 2018, Article ID 6409389, 15 pages, 2018.

[60] W.-T. Zhang, Z. Jin, F. Luo, L. Zhang, Y.-W. Zeng, and J.-S. Han, "Evidence from brain imaging with fMRI supporting functional specificity of acupoints in humans," Neuroscience Letters, vol. 354, no. 1, pp. 50-53, 2004.

[61] V. Napadow, N. Makris, J. Liu, N. W. Kettner, K. K. Kwong, and K. K. S. Hui, "Effects of electroacupuncture versus manual acupuncture on the human brain as measured by fMRI," Human Brain Mapping, vol. 24, no. 3, pp. 193-205, 2005.

[62] M.-T. Wu, J.-C. Hsieh, J. Xiong et al., "Central nervous pathway for acupuncture stimulation: localization of processing with functional MR imaging of the brain-preliminary experience,” Radiology, vol. 212, no. 1, pp. 133-141, 1999.

[63] B. Ellenbroek and J. Youn, "Rodent models in neuroscience research: is it a rat race?" Disease Models \& Mechanisms, vol. 9, no. 10, pp. 1079-1087, 2016.

[64] A. H. D. Mello, R. D. B. Schraiber, and M. P. D. S. Goldim, "Omega-3 fatty acids attenuate brain alterations in high-fat diet-induced obesity model," Molecular Neurobiology, vol. 2, pp. 1-12, 2019.

[65] C. Rodríguez-Rodríguez, N. Torres, J. A. Gutiérrez-Uribe et al., "The effect of isorhamnetin glycosides extracted from Opuntia ficus-indica in a mouse model of diet induced obesity," Food \& Function, vol. 6, no. 3, pp. 805-815, 2015.

[66] S. Kondo, J.-z. Xiao, T. Satoh et al., "Antiobesity effects ofBifidobacterium breveStrain B-3 supplementation in a mouse model with high-fat diet-induced obesity," Bioscience Biotechnology and Biochemistry, vol. 74, no. 8, pp. 16561661, 2010.

[67] R. Crescenzo, F. Bianco, I. Falcone, P. Coppola, G. Liverini, and S. Iossa, "Increased hepatic de novo lipogenesis and mitochondrial efficiency in a model of obesity induced by diets rich in fructose," European Journal of Nutrition, vol. 52, no. 2, pp. 537-545, 2013.

[68] C. Marques, M. Meireles, S. Norberto et al., "High-fat dietinduced obesity rat model: a comparison between wistar and sprague-dawley rat," Adipocyte, vol. 5, no. 1, pp. 11-21, 2016.

[69] C. Torres-Fuentes, H. Schellekens, T. G. Dinan, and J. F. Cryan, "The microbiota-gut-brain axis in obesity," The Lancet Gastroenterology \& Hepatology, vol. 2, no. 10, pp. 747-756, 2017.

[70] J. K. Yee, W.-N. P. Lee, G. Han, M. G. Ross, and M. Desai, "Organ-specific alterations in fatty acid de novo synthesis and desaturation in a rat model of programmed obesity," Lipids in Health and Disease, vol. 10, no. 1, p. 72, 2011.

[71] K. Takemori, T. Kimura, N. Shirasaka, T. Inoue, K. Masuno, and H. Ito, "Food restriction improves glucose and lipid metabolism through Sirt1 expression: a study using a new rat 
model with obesity and severe hypertension," Life Sciences, vol. 88, no. 25, pp. 1088-1094, 2011.

[72] A. Marco, M. Schroeder, and A. Weller, "Feeding and reward: ontogenetic changes in an animal model of obesity," Neuropharmacology, vol. 62, no. 8, pp. 2447-2454, 2012.

[73] T. W. Castonguay, W. J. Hartman, E. A. Fitzpatrick, and J. S. Stern, "Dietary self-selection and the Zucker rat," Journal of Nutrition, vol. 112, no. 4, pp. 796-800, 1982.

[74] K. Takaya, Y. Ogawa, N. Isse et al., "Molecular cloning of rat leptin receptor isoform complementary DNAs-identification of a missense mutation in Zucker fatty ( $\mathrm{fa} / \mathrm{fa}$ ) rats," Biochemical and Biophysical Research Communications, vol. 225, no. 1, pp. 75-83, 1996.

[75] J. L. Buckley and E. B. Rasmussen, "Obese and lean Zucker rats demonstrate differential sensitivity to rates of food reinforcement in a choice procedure," Physiology \& Behavior, vol. 108, pp. 19-27, 2012.

[76] X. Ma, V. T. Pham, H. Mori, O. A. Macdougald, Y. M. Shah, and P. F. Bodary, "Iron elevation and adipose tissue remodeling in the epididymal depot of a mouse model of polygenic obesity," PLoS One, vol. 12, no. 6, Article ID e0179889, 2017.

[77] Y. F. Dong, L. Liu, K. Kataoka et al., "Aliskiren prevents cardiovascular complications and pancreatic injury in a mouse model of obesity and type 2 diabetes," Diabetologia, vol. 53, no. 1, pp. 180-191, 2010.

[78] A. Dey, S. Hao, J. R. Erion, M. Wosiski-Kuhn, and A. M. Stranahan, "Glucocorticoid sensitization of microglia in a genetic mouse model of obesity and diabetes," Journal of Neuroimmunology, vol. 269, no. 1-2, pp. 20-27, 2014.

[79] L. Ji-Yeon, M. Heike, and G. Oksana, "Loss of cytokineSTAT5 signaling in the CNS and pituitary gland alters energy balance and leads to obesity," PLoS One, vol. 3, no. 2, Article ID e1639, 2008.

[80] B. Zhong, J. Rubinstein, S. Ma, and D. H. Wang, "Knockout of TRPV1 exacerbates left ventricular diastolic dysfunction induced by A high-fat diet in mice," Cardiovascular \& Haematological Disorders-Drug Targets, vol. 18, no. 3, pp. 215-223, 2008.

[81] M. Lu, Y. He, M. Gong et al., "Role of neuro-immune crosstalk in the anti-obesity effect of electro-acupuncture," Frontiers in Neuroscience, vol. 14, p. 151, 2020.

[82] L. L. Xie, Y. L. Zhao, J. Yang et al., "Electroacupuncture prevents osteoarthritis of high-fat diet-induced obese rats," BioMed Research International, vol. 2020, Article ID 9380965, 16 pages, 2020.

[83] H. Wang, Q. Wang, C. Liang et al., "Acupuncture regulating gut microbiota in abdominal obese rats induced by high-fat diet," Evidence-Based Complementary and Alternative Medicine, vol. 2019, Article ID 4958294, 12 pages, 2019.

[84] X. Jie, X. Li, J. Q. Song, D. Wang, and J. H. Wang, "Antiinflammatory and autonomic effects of electroacupuncture in a rat model of diet-induced obesity," Acupuncture in Medicine, vol. 36, no. 2, 2018.

[85] J. Leng, F. Xiong, J. Yao et al., "Electroacupuncture reduces weight in diet-induced obese rats via hypothalamic Tsc1 promoter demethylation and inhibition of the activity of mTORC1 signaling pathway," Evidence-based Complementary and Alternative Medicine, vol. 2018, Article ID 3039783, 10 pages, 2018.

[86] M. Gong, C. Cao, F. Chen et al., "Electroacupuncture attenuates hepatic lipid accumulation via AMP-activated protein kinase (AMPK) activation in obese rats," Acupuncture in Medicine, vol. 34, no. 3, pp. 209-214, 2016.
[87] X. Liu, J.-F. He, Y.-T. Qu et al., "Electroacupuncture improves insulin resistance by reducing neuroprotein $\mathrm{Y} /$ Agouti-Related protein levels and inhibiting expression of protein tyrosine phosphatase $1 \mathrm{~B}$ in diet-induced obese rats," Journal of Acupuncture and Meridian Studies, vol. 9, no. 2, pp. 58-64, 2016.

[88] J. Xu, L. Chen, L. Tang et al., "Electroacupuncture inhibits weight gain in diet-induced obese rats by activating hypothalamicLKB1-AMPK signaling," BMC Complementary and Alternative Medicine, vol. 15, no. 1, p. 147, 2015.

[89] J. Liu, H. Jin, R. D. Foreman et al., "Chronic electrical stimulation at acupoints reduces body weight and improves blood glucose in obese rats via autonomic pathway," Obesity Surgery, vol. 25, no. 7, pp. 1209-1216, 2015.

[90] W. Chorng-Kai and L. Tzung-Yan, "Electroacupuncture decreases the leukocyte infiltration to white adipose tissue and attenuates inflammatory response in high fat diet-induced obesity rats," Evidence-Based Complementray and Alternative Medicine, vol. 2014, Article ID 473978, 11 pages, 2014.

[91] R. Yan, X. Liu, J. Bai, J. Yu, and J. Gu, "Influence of catgut implantation at acupoints on leptin and insulin resistance in simple obesity rats," Journal of Traditional Chinese Medicine, vol. 32, no. 3, pp. 477-481, 2012.

[92] M. Gong, X. Wang, Z. Mao, Q. Shao, X. Xiang, and B. Xu, "Effect of electroacupuncture on leptin resistance in rats with diet-induced obesity," American Journal of Chinese Medicine, vol. 40, no. 3, pp. 511-520, 2012.

[93] J.-L. Guan, Q.-P. Wang, H. Kageyama et al., "Synaptic interactions between ghrelin- and neuropeptide Y-containing neurons in the rat arcuate nucleus," Peptides, vol. 24, no. 12, pp. 1921-1928, 2003.

[94] D. R. Tian, X. D. Li, F. Wang et al., "Up-regulation of the expression of cocaine and amphetamine-regulated transcript peptide by electroacupuncture in the arcuate nucleus of dietinduced obese rats," Neuroscience Letters, vol. 383, no. 1-2, pp. 17-21, 2005.

[95] Y. Zhang, L. Xu, M. Ding, G. Su, and Y. Zhao, "Anti-obesity effect of garlic oil on obese rats via Shenque point administration," Journal of Ethnopharmacology, vol. 231, pp. 486493, 2019.

[96] D. Luo, L. Liu, F. X. Liang, Z. M. Yu, and R. Chen, "Electroacupuncture: a feasible Sirt1 promoter which modulates metainflammation in diet-induced obesity rats," EvidenceBased Complementary and Alternative Medicine, vol. 2018, Article ID 5302049, 10 pages, 2018.

[97] M. C. Pontes, L. C. Heck, and J. C. Coelho, "Behavioral and biochemical effects of pharmacopuncture (ST 36 and ST 25) in obese rats," BMC Complementary and Alternative Medicine, vol. 15, no. 1, p. 297, 2015.

[98] T. Shiraishi, M. Onoe, T. Kojima, Y. Sameshima, and T. Kageyama, "Effects of auricular stimulation on feedingrelated hypothalamic neuronal activity in normal and obese rats," Brain Research Bulletin, vol. 36, no. 2, pp. 141-148, 1995.

[99] S. Asamoto and C. Takeshige, "Activation of the satiety center by auricular acupuncture point stimulation," Brain Research Bulletin, vol. 29, no. 2, pp. 157-164, 1992.

[100] C. Inprasit, Y. C. Huang, and Y. W. Lin, "Evidence for acupoint catgut embedding treatment and TRPV1 gene deletion increasing weight control in murine model," International Journal of Molecular Medicine, vol. 45, no. 3, pp. 779-792, 2020. 
[101] S.-F. Lu, Y.-X. Tang, T. Zhang et al., "Electroacupuncture reduces body weight by regulating fat browning-related proteins of adipose tissue in HFD-induced obese mice," Frontiers in Psychiatry, vol. 10, p. 353, 2019.

[102] Y. C. Si, W. N. Miao, J. Y. He, L. Chen, Y. L. Wang, and W. J. Ding, "Regulating gut flora dysbiosis in obese mice by electroacupuncture," American Journal of Chinese Medicine, pp. 1-17, 2018, inpress.

[103] W. Shen, Y. Wang, S.-F. Lu et al., "Acupuncture promotes white adipose tissue browning by inducing UCP1 expression on DIO mice," BMC Complementary and Alternative Medicine, vol. 14, no. 1, p. 501, 2014.

[104] X.-Y. Huang, L. Zhang, J. Sun, N.-G. Xu, and W. Yi, "Acupuncture alters expression of insulin signaling related molecules and improves insulin resistance in OLETF rats," Evidence-based Complementary and Alternative Medicine, vol. 2016, no. 1, Article ID 9651592, 7 pages, 2016.

[105] H. Nakamura, T. Ishigami, Y. Kawase et al., "Effects of acupuncture stimulation on blood glucose concentration in the Otsuka Long-Evans Tokushima Fatty (OLETF) rat, an animal model for type-2 diabetes mellitus," Medical science monitor basic research, vol. 20, pp. 70-75, 2014.

[106] J. J. T. Liaw and P. V. Peplow, "Effects of electroacupuncture on pro-/anti-inflammatory adipokines in serum and adipose tissue in lean and diet-induced obese rats," Journal of Acupuncture and Meridian Studies, vol. 9, no. 2, pp. 65-72, 2016.

[107] J. J. T. Liaw and P. V. Peplow, "Differential effect of electroacupuncture on inflammatory adipokines in two rat models of obesity," Journal of Acupuncture and Meridian Studies, vol. 9, no. 4, pp. 183-190, 2016.

[108] J. J. T. Liaw and P. V. Peplow, "Effect of electroacupuncture on inflammation in the obese zucker fatty rat model of metabolic syndrome," Journal of Acupuncture and Meridian Studies, vol. 9, no. 2, pp. 73-79, 2016.

[109] S.-P. Fu, H. Hong, S.-F. Lu et al., "Genome-wide regulation of electro-acupuncture on the neural Stat5-loss-induced obese mice," PLoS One, vol. 12, no. 8, Article ID e0181948, 2017.

[110] C.-K. Wen and T.-Y. Lee, "Electroacupuncture prevents white adipose tissue inflammation through modulation of hypoxia-inducible factors- $1 \alpha$-dependent pathway in obese mice," BMC Complementary and Alternative Medicine, vol. 15, no. 1, p. 452, 2015.

[111] B. Ji, J. Hu, and S. Ma, "Effects of electroacupuncture Zusanli (ST36) on food intake and expression of POMC and TRPV1 through afferents-medulla pathway in obese prone rats," Peptides, vol. 40, pp. 188-194, 2013.

[112] M. Choowanthanapakorn, K.-W. Lu, J. Yang, C.-L. Hsieh, and Y.-W. Lin, "Targeting TRPV1 for body weight control using TRPV1-/- mice and electroacupuncture," Scientific Reports, vol. 5, no. 1, Article ID 17366, 2015.

[113] W. Fei Wang, D. R. Tian, P. Tso, and J. S. Han, “Arcuate nucleus of hypothalamus is involved in mediating the satiety effect of electroacupuncture in obese rats," Peptides, vol. 32, no. 12, pp. 2394-2399, 2011.

[114] P. V. Peplow, "Repeated electroacupuncture in obese Zucker diabetic fatty rats: adiponectin and leptin in serum and adipose tissue," Journal of Acupuncture and Meridian Studies, vol. 8, no. 2, pp. 66-70, 2015.

[115] R. B. Ceddia, H. A. Koistinen, J. R. Zierath, and G. Sweeney, "Analysis of paradoxical observations on the association between leptin and insulin resistance," The FASEB Journal, vol. 16, no. 10, pp. 1163-1176, 2002.
[116] B. C. Finger, T. G. Dinan, and J. F. Cryan, "Behavioral satiety sequence in a genetic mouse model of obesity," Behavioural Pharmacology, vol. 22, no. 7, pp. 624-632, 2011.

[117] W. T. Zhou, S. Y. Jia, and Y. Q. Zhang, "Pathological changes in internal organs after blocking low hydraulic resistance channels along the stomach meridian in pigs," EvidenceBased Complementary and Alternative Medicine, vol. 2013, Article ID 935687, 12 pages, 2013.

[118] K. Deisseroth, "Optogenetics: 10 years of microbial opsins in neuroscience," Nature Neuroscience, vol. 18, no. 9, pp. 1213-1225, 2015.

[119] D. Zelena, K. Demeter, J. Haller, and D. Balázsfi, “Considerations for the use of virally delivered genetic tools for invivo circuit analysis and behavior in mutant mice: a practical guide to optogenetics," Behavioural Pharmacology, vol. 28, no. 8, pp. 598-609, 2017.

[120] S. R. Boomhower, E. B. Rasmussen, and T. S. Doherty, "Impulsive-choice patterns for food in genetically lean and obese Zucker rats," Behavioural Brain Research, vol. 241, no. 2, pp. 214-221, 2013.

[121] D. P. Jarmolowicz, J. L. Hudnall, L. Hale et al., "Delay discounting as impaired valuation: delayed rewards in an animal obesity model," Journal of the Experimental Analysis of Behavior, vol. 108, no. 2, pp. 171-183, 2017.

[122] D. P. Jarmolowicz, J. B. C. Cherry, D. D. Reed et al., "Robust relation between temporal discounting rates and body mass," Appetite, vol. 78, pp. 63-67, 2014.

[123] L. Zhang and I. Rashad, "Obesity and time preference: the health consequences of discounting the future," Journal of Biosocial Science, vol. 40, no. 1, pp. 97-113, 2008.

[124] L. D. Pound, P. Kievit, and K. L. Grove, "The nonhuman primate as a model for type 2 diabetes," Current Opinion in Endocrinology Diabetes and Obesity, vol. 21, no. 2, pp. 89-94, 2014.

[125] P. B. Higgins, R. A. Bastarrachea, J. C. Lopez-Alvarenga et al., "Eight week exposure to a high sugar high fat diet results in adiposity gain and alterations in metabolic biomarkers in baboons (Papio hamadryas sp.)," Cardiovascular Diabetology, vol. 9, no. 1, p. 71, 2010.

[126] A. A. Bremer, K. L. Stanhope, J. L. Graham et al., "Fructosefed rhesus monkeys: a nonhuman primate model of insulin resistance, metabolic syndrome, and type 2 diabetes," Clinical and Translational Science, vol. 4, no. 4, pp. 243-252, 2011.

[127] J. Ma, A. L. Prince, D. Bader et al., "High-fat maternal diet during pregnancy persistently alters the offspring microbiome in a primate model," Nature Communications, vol. 5, no. 1, p. 3889, 2014.

[128] Y. Guo, Z. Wang, L. Chen et al., "Diet induced maternal obesity affects offspring gut microbiota and persists into young adulthood," Food \& Function, vol. 9, no. 8, pp. 4317-4327, 2018.

[129] P. J. Asquith and P. J. Asquith, "Provisioning and the study of free-ranging primates: history, effects, and prospects," American Journal of Physical Anthropology, vol. 32, no. S10, pp. 129-158, 1989.

[130] S. M. Schwartz and J. W. Kemnitz, "Age- and gender-related changes in body size, adiposity, and endocrine and metabolic parameters in free-ranging rhesus macaques," American Journal of Physical Anthropology, vol. 89, no. 1, pp. 109-121, 1992.

[131] J. W. Kemnitz, "Obesity in macaques: spontaneous and induced," Advances in Veterinary Science \& Comparative Medicine, vol. 28, no. 4, pp. 81-114, 1984. 
[132] C. Yang, O. Fumiko, Y. Takashi, and Y. Yasuhiro, "Relationship between body weight and hematological and serum biochemical parameters in female cynomolgus monkeys (Macaca fascicularis)," Experimental Animals, vol. 51, no. 2, pp. 125-131, 2002.

[133] R. V. Considine, M. K. Sinha, M. L. Heiman et al., "Serum immunoreactive-leptin concentrations in normal-weight and obese humans," New England Journal of Medicine, vol. 334, no. 5, pp. 292-295, 1996.

[134] R. A. Harris, C. E. Alcott, E. L. Sullivan et al., "Genomic variants associated with resistance to high fat diet induced obesity in a primate model," Scientific Reports, vol. 6, no. 1, Article ID 36123, 2016.

[135] A. E. Locke, B. Kahali, S. I. Berndt et al., "Genetic studies of body mass index yield new insights for obesity biology," Nature, vol. 518, no. 7538, pp. 197-206, 2015.

[136] J. Sallet, R. B. Mars, M. P. Noonan et al., "The organization of dorsal frontal cortex in humans and macaques," Journal of Neuroscience, vol. 33, no. 30, pp. 12255-12274, 2013.

[137] T. A. Chaplin, H.-H. Yu, J. G. M. Soares, R. Gattass, and M. G. P. Rosa, "A conserved pattern of differential expansion of cortical areas in simian primates," Journal of Neuroscience, vol. 33, no. 38, pp. 15120-15125, 2013.

[138] J. Jelsing, A. Hay-Schmidt, T. Dyrby, R. Hemmingsen, H. B. M. Uylings, and B. Pakkenberg, "The prefrontal cortex in the Göttingen minipig brain defined by neural projection criteria and cytoarchitecture," Brain Research Bulletin, vol. 70, no. 4-6, pp. 322-336, 2006.

[139] H. Barbas and D. N. Pandya, "Architecture and frontal cortical connections of the premotor cortex (area 6) in the rhesus monkey," The Journal of Comparative Neurology, vol. 256, no. 2, pp. 211-228, 1987.

[140] D. C. Van Essen, W. T. Newsome, J. H. R. Maunsell, and J. L. Bixby, "The projections from striate cortex (V1) to areas $\mathrm{V} 2$ and V3 in the macaque monkey: asymmetries, areal boundaries, and patchy connections," The Journal of Comparative Neurology, vol. 244, no. 4, pp. 451-480, 1986.

[141] D. Zhang, L. Guo, D. Zhu et al., "Diffusion tensor imaging reveals evolution of primate brain architectures," Brain Structure and Function, vol. 218, no. 6, pp. 1429-1450, 2013.

[142] P. L. Croxson, S. J. Forkel, L. Cerliani, and M. Thiebaut de Schotten, "Structural variability across the primate brain: a cross-species comparison," Cerebral Cortex, vol. 28, no. 11, pp. 3829-3841, 2018.

[143] E. R. Magden, R. L. Haller, E. J. Thiele, S. J. Buchl, S. P. Lambeth, and S. J. Schapiro, "Acupuncture as an adjunct therapy for osteoarthritis in chimpanzees (Pan troglodytes)," Journal of the American Association for Laboratory Animal, vol. 52, no. 4, pp. 475-480, 2013.

[144] F. Zhao, X. Fan, R. Grondin et al., "Improved methods for electroacupuncture and electromyographic recordings in normal and parkinsonian rhesus monkeys," Journal of Neuroscience Methods, vol. 192, no. 2, pp. 199-206, 2010.

[145] C. N. Ross, M. L. Power, J. M. Artavia, and S. D. Tardif, "Relation of food intake behaviors and obesity development in young common marmoset monkeys," Obesity, vol. 21, no. 9, pp. 1891-1899, 2013.

[146] C. L. Adam, P. A. Findlay, R. P. Aitken, J. S. Milne, and J. M. Wallace, "In vivo changes in central and peripheral insulin sensitivity in a large animal model of obesity," Endocrinology, vol. 153, no. 7, pp. 3147-3157, 2012.

[147] E. Roura, S.-J. Koopmans, J.-P. Lallès et al., "Critical review evaluating the pig as a model for human nutritional physiology," Nutrition Research Reviews, vol. 29, no. 1, pp. 60-90, 2016.

[148] D. Val-Laillet, S. Layec, S. Guérin, P. Meurice, and C.-H. Malbert, "Changes in brain activity after a diet-induced obesity,” Obesity, vol. 19, no. 4, pp. 749-756, 2011.

[149] A. D. Lassaletta, L. M. Chu, M. P. Robich et al., "Overfed Ossabaw swine with early stage metabolic syndrome have normal coronary collateral development in response to chronic ischemia," Basic Research in Cardiology, vol. 107, no. 2, p. 243, 2012.

[150] R. G. Toedebusch, M. D. Roberts, K. D. Wells et al., "Unique transcriptomic signature of omental adipose tissue in Ossabaw swine: a model of childhood obesity," Physiological Genomics, vol. 46, no. 10, pp. 362-375, 2014.

[151] P. Sauleau, E. Lapouble, D. Val-Laillet, and C.-H. Malbert, "The pig model in brain imaging and neurosurgery," Animal, vol. 3, no. 8, pp. 1138-1151, 2009.

[152] D. Val-Laillet, "Review: impact of food, gut-brain signals and metabolic status on brain activity in the pig model: 10 years of nutrition research using in vivo brain imaging," Animal, vol. 13, no. 11, pp. 2699-2713, 2019.

[153] J. Weickenmeier, M. Kurt, E. Ozkaya, M. Wintermark, K. B. Pauly, and E. Kuhl, "Magnetic resonance elastography of the brain: a comparison between pigs and humans," Journal of the Mechanical Behavior of Biomedical Materials, vol. 77, pp. 702-710, 2018.

[154] M. A. Hofman, "Size and shape of the cerebral cortex in mammals," Brain, Behavior and Evolution, vol. 27, no. 1, pp. 28-40, 1985.

[155] S. L. Craner and R. H. Ray, "Somatosensory cortex of the neonatal pig: I. Topographic organization of the primary somatosensory cortex (SI)," The Journal of Comparative Neurology, vol. 306, no. 1, pp. 24-38, 1991.

[156] C. R. Bjarkam, A. N. Glud, D. Orlowski, J. C. H. Sørensen, and N. Palomero-Gallagher, "The telencephalon of the Göttingen minipig, cytoarchitecture and cortical surface anatomy," Brain Structure and Function, vol. 222, no. 5, pp. 2093-2114, 2017.

[157] C. M. J. Mentzel, F. Alkan, H. Keinicke et al., "Joint profiling of miRNAs and mRNAs reveals miRNA mediated gene regulation in the göttingen minipig obesity model," PLoS One, vol. 11, no. 11, Article ID e0167285, 2016.

[158] A. Barbero, S. Astiz, C. Ovilo et al., "Prenatal programming of obesity in a swine model of leptin resistance: modulatory effects of controlled postnatal nutrition and exercise," Journal of Developmental Origins of Health and Disease, vol. 5, no. 3, pp. 248-258, 2014.

[159] Y. Gautier, I. Luneau, N. Coquery et al., "Maternal Western diet during gestation and lactation modifies adult offspring's cognitive and hedonic brain processes, behavior, and metabolism in Yucatan minipigs," Faseb Journal Official Publication of the Federation of American Societies for Experimental Biology, Article ID fj201701541, 2018, inpress.

[160] D. Val-Laillet, A. Kanzari, S. Guérin, G. Randuineau, and N. Coquery, "A maternal Western diet during gestation and lactation modifies offspring's microglial cell density and morphology in the hippocampus and prefrontal cortex in Yucatan minipigs," Neuroscience Letters, vol. 739, Article ID 135395, 2020.

[161] L. J. A. Kogelman, H. N. Kadarmideen, T. Mark et al., “An F2 pig resource population as a model for genetic studies of obesity and obesity-related diseases in humans: design and genetic parameters," Frontiers in Genetics, vol. 4, no. 1, p. 29, 2013. 
[162] J. Wei, H. Ouyang, Y. Wang et al., "Characterization of a hypertriglyceridemic transgenic miniature pig model expressing human apolipoprotein CIII," FEBS Journal, vol. 279, no. 1, pp. 91-99, 2012.

[163] X. Tang, G. Wang, X. Liu et al., "Overexpression of porcine lipoprotein-associated phospholipase A 2 in swine," Biochemical and Biophysical Research Communications, vol. 465, no. 3, pp. 507-511, 2015.

[164] S. A. Ferguson, N. V. Gopee, M. G. Paule, and P. C. Howard, "Female mini-pig performance of temporal response differentiation, incremental repeated acquisition, and progressive ratio operant tasks," Behavioural Processes, vol. 80, no. 1, pp. 28-34, 2009.

[165] S. Held, J. Baumgartner, A. Kilbride, R. W. Byrne, and M. Mendl, "Foraging behaviour in domestic pigs (Sus scrofa): remembering and prioritizing food sites of different value," Animal Cognition, vol. 8, no. 2, pp. 114-121, 2005.

[166] G. P. Smith, "Accumbens dopamine mediates the rewarding effect of orosensory stimulation by sucrose," Appetite, vol. 43, no. 1, pp. 11-13, 2004.

[167] C. Clouard, M. Jouhanneau, M.-C. Meunier-Salaün, C.-H. Malbert, and D. Val-Laillet, "Exposures to conditioned flavours with different hedonic values induce contrasted behavioural and brain responses in pigs," PLoS One, vol. 7, no. 5, Article ID e37968, 2012.

[168] J. A. Gottfried, J. O’Doherty, and R. J. Dolan, “Appetitive and aversive olfactory learning in humans studied using eventrelated functional magnetic resonance imaging," Journal of Neuroscience, vol. 22, no. 24, pp. 10829-10837, 2002.

[169] M. Oostindjer, J. E. Bolhuis, H. van den Brand, E. Roura, and B. Kemp, "Prenatal flavor exposure affects growth, health and behavior of newly weaned piglets," Physiology \& Behavior, vol. 99, no. 5, pp. 579-586, 2010.

[170] J. A. Mennella and G. K. Beauchamp, "The human infants' response to vanilla flavors in mother's milk and formula," Infant Behavior and Development, vol. 19, no. 1, pp. 13-19, 1996.

[171] P. Chandler, J. Viana, K. Oswald, P. Wauford, and M. Boggiano, "Feeding response to melanocortin agonist predicts preference for and obesity from a high-fat diet," Physiology \& Behavior, vol. 85, no. 2, pp. 221-230, 2005.

[172] J. H. Lin and R. Panzer, "Acupuncture for reproductive disorders," Problems in Veterinary Medicine, vol. 4, no. 1, p. 155, 1992.

[173] Y. C. Hwang and E. M. Jenkins, "Effect of acupuncture on young pigs with induced enteropathogenic Escherichia coli diarrhea," American Journal of Veterinary Research, vol. 49, no. 9, pp. 1641-1643, 1988.

[174] K. Takeishi, M. Horiuchi, H. Kawaguchi et al., "Acupuncture improves sleep conditions of minipigs representing diurnal animals through an anatomically similar point to the acupoint (GV20) effective for humans," Evidence-Based Complementary and Alternative Medicine, vol. 2012, Article ID 472982, 6 pages, 2012.

[175] B. Faramarzi, D. Lee, K. May, and F. Dong, "Response to acupuncture treatment in horses with chronic laminitis," The Canadian Veterinary Journal $=L$ a revue veterinaire canadienne, vol. 58, no. 8, pp. 823-827, 2017.

[176] A. Pons, S. Canfrán, J. Benito, R. Cediel-Algovia, and I. A. Gómez de Segura, "Effects of dexmedetomidine administered at acupuncture point GV20 compared to intramuscular route in dogs," Journal of Small Animal Practice, vol. 58, no. 1, pp. 23-28, 2017.
[177] S. Neof, L. Spl, J. Jgf, H. D. Coutinho, and F. S. Possebon, "Effect of acupuncture on pain and quality of life in canine neurological and musculoskeletal diseases," The Canadian Veterinary Journal = La revue veterinaire canadienne, vol. 58, no. 9, p. 941, 2017.

[178] X. Zhang, H. Chen, and D. Val-Laillet, "Hypothesis paper: electroacupuncture targeting the gut-brain axis to modulate neurocognitive determinants of eating behavior-toward a proof of concept in the obese minipig model," Eating and Weight Disorders, vol. 26, no. 1, 2021.

[179] E. P. Kirk, J. E. Donnelly, B. K. Smith et al., "Minimal resistance training improves daily energy expenditure and fat oxidation," Medicine \& Science in Sports \& Exercise, vol. 41, no. 5, pp. 1122-1129, 2009.

[180] C. K. Roberts, D. M. Croymans, N. Aziz, A. W. Butch, and C. C. Lee, "Resistance training increases SHBG in overweight/obese, young men," Metabolism, vol. 62, no. 5, pp. $725-733,2013$.

[181] C. A. Smith, S. de Lacey, M. Chapman et al., "Effect of acupuncture vs sham acupuncture on live births among women undergoing in vitro fertilization," Journal of the American Medical Association, vol. 319, no. 19, pp. 19901998, 2018.

[182] K. K. S. Hui, J. Liu, N. Makris et al., "Acupuncture modulates the limbic system and subcortical gray structures of the human brain: evidence from fMRI studies in normal subjects," Human Brain Mapping, vol. 9, no. 1, pp. 13-25, 2000.

[183] E. Frangos, J. Ellrich, and B. R. Komisaruk, "Non-invasive access to the vagus nerve central projections via electrical stimulation of the external ear: fMRI evidence in humans," Brain Stimulation, vol. 8, no. 3, pp. 624-636, 2015.

[184] M. J. Mason, "Of mice, moles and Guinea pigs: functional morphology of the middle ear in living mammals," Hearing Research, vol. 301, pp. 4-18, 2013.

[185] H. Li, J. B. Zhang, C. Xu et al., "Effects and mechanisms of auricular vagus nerve stimulation on high-fat-diet--induced obese rats," Nutrition, vol. 31, no. 11-12, pp. 1416-1422, 2015.

[186] M. Hoffstetter, F. Lugauer, S. Kundu et al., "Middle ear of human and pig: a comparison of structures and mechanics," Biomedizinische Technik/Biomedical Engineering, vol. 56, no. 3, pp. 159-165, 2011.

[187] M. N. Coleman and C. F. Ross, "Primate auditory diversity and its influence on hearing performance," The Anatomical Record, vol. 281A, no. 1, pp. 1123-1137, 2004.

[188] A. Ishikawa and S. Okuno, "Fine mapping and candidate gene search of quantitative trait loci for growth and obesity using mouse intersubspecific subcongenic intercrosses and exome sequencing," PLoS One, vol. 9, no. 9, Article ID e113233, 2014.

[189] K. Gerozissis, M. Orosco, C. Rouch, and S. Nicolaidis, "Basal and hyperinsulinemia-induced immunoreactive hypothalamic insulin changes in lean and genetically obese Zucker rats revealed by microdialysis," Brain Research, vol. 611, no. 2, pp. 258-263, 1993.

[190] C. K. Kim, A. Adhikari, and K. Deisseroth, "Integration of optogenetics with complementary methodologies in systems neuroscience," Nature Reviews Neuroscience, vol. 18, no. 4, pp. 222-235, 2017.

[191] F. Zhang, V. Gradinaru, A. R. Adamantidis et al., "Optogenetic interrogation of neural circuits: technology for probing mammalian brain structures," Nature Protocols, vol. 5, no. 3, pp. 439-456, 2010. 
[192] F. Zhang, L.-P. Wang, M. Brauner et al., "Multimodal fast optical interrogation of neural circuitry," Nature, vol. 446, no. 7136, pp. 633-639, 2007.

[193] H. E. Covington, M. K. Lobo, I. Maze et al., "Antidepressant effect of optogenetic stimulation of the medial prefrontal cortex," Journal of Neuroscience, vol. 30, no. 48, pp. 16082-16090, 2010.

[194] C. Clouard, M. C. Meunier-Salaün, and D. Val-Laillet, "Food preferences and aversions in human health and nutrition: how can pigs help the biomedical research?" Animal, vol. 6, no. 1, pp. 118-136, 2012.

[195] B. M. Appelhans, K. Woolf, S. L. Pagoto, K. L. Schneider, M. C. Whited, and R. Liebman, "Inhibiting food reward: delay discounting, food reward sensitivity, and palatable food intake in overweight and obese women," Obesity, vol. 19, no. 11, pp. 2175-2182, 2011.

[196] M. Ochoa, C.-H. Malbert, P. Meurice, and D. Val-Laillet, "Effects of chronic consumption of sugar-enriched diets on brain metabolism and insulin sensitivity in adult yucatan minipigs," PLoS One, vol. 11, no. 9, Article ID e0161228, 2016.

[197] D. Val-Laillet, M. Besson, S. Guérin et al., “A maternal Western diet during gestation and lactation modifies offspring's microbiota activity, blood lipid levels, cognitive responses, and hippocampal neurogenesis in Yucatan pigs," The FASEB Journal, vol. 31, no. 5, pp. 2037-2049, 2017.

[198] G. Srivastava and C. M. Apovian, "Current pharmacotherapy for obesity," Nature Reviews Endocrinology, vol. 14, no. 1, pp. 12-24, 2018.

[199] D. H. Bessesen and L. F. Van Gaal, "Progress and challenges in anti-obesity pharmacotherapy," The Lancet Diabetes \& Endocrinology, vol. 6, no. 3, pp. 237-248, 2018.

[200] A. Chung, L. Bui, and E. Mills, "Adverse effects of acupuncture. Which are clinically significant?" Canadian Family Physician, vol. 49, pp. 985-989, 2003. 